\title{
Experimental and numerical investigation of the dynamics of a coalesced oscillating bubble near a free surface
}

\author{
Rui Han ${ }^{1,2}$, Longbin Tao ${ }^{1,2 *}$, A-Man Zhang ${ }^{1}$, Shuai Li ${ }^{1}$ \\ 1) College of Shipbuilding Engineering, Harbin Engineering University, Harbin 150001, China \\ 2) Department of Naval Architecture, Ocean and Marine Engineering, University of Strathclyde, Glasgow G4 \\ OLZ, United Kingdom
}

Understanding the dynamics of oscillating bubbles beneath a free surface is crucial to many practical applications including airgun-bubble clusters, underwater explosions, etc. In this paper, an experimental and numerical study of the dynamic behaviors of a coalesced bubble near a free surface is conducted, which shows quite different physical features from single bubble dynamics. Firstly, two similar sized underwater discharge bubbles are generated simultaneously beneath a free surface and their complex interactions are experimentally studied with high-speed photography imaging. A strong interaction between two bubbles and the subsequent coalescence are observed when the initial distance between two bubbles is smaller than the maximum equivalent bubble radius. Secondly, both axisymmetric and three-dimensional (3D) boundary integral models are used to simulate the precoalescence and post-coalescence of two bubbles. The results obtained by the two models agree well in axisymmetric conditions. The essential physical phenomena in representative experiments are well reproduced by the present 3D model. The pressure field is calculated by the auxiliary function method, which helps to reveal the underlying mechanisms of bubble collapse patterns and jetting behaviors. A parametric study reveals the dependence of the coalesced bubble dynamics and free surface motion on the governing dimensionless quantities.

Key words: bubble dynamics; bubble coalescence; free surface

\footnotetext{
*Email: longbin.tao@strath.ac.uk
} 


\section{INTRODUCTION}

The dynamic behaviors of multiple bubbles beneath a free surface are of great importance in various natural process and engineering applications, including underwater explosion near a free surface (Brett and Krelle, 2018; Cui et al., 2016b; Li et al., 2018a; Wang et al., 2018a), airgun clusters for seabed exploration (Cox et al., 2004; de Graaf et al., 2014), cavitation (Long et al., 2018; Wang et al., 2017; Wang et al., 2018b), laser-induced forward transfer (Turkoz et al., 2018a; Turkoz et al., 2018b), bubble damage on tissue and cells (Chen et al., 2009; Curtiss et al., 2013; Liu et al., 2018) and bubble drag reduction (Verschoof et al., 2016).

When a single oscillating bubble is initiated near a free surface, the non-uniform pressure gradient surrounding the bubble often induces non-spherical collapse of the bubble. Generally, a downward liquid jet is formed inside the collapsing bubble, while the free surface produces an upward spike (Blake et al., 1987). In addition, an upward jet would form from the bubble bottom if the bubble subjects to a relatively large buoyancy effect (Klaseboer et al., 2005b; Wang et al., 1996b; Zhang et al., 2015a). The dynamics of a single bubble beneath a free surface have been studied in a great detail in the past several decades (Dadvand et al., 2012; Kannan et al., 2018; Pearson et al., 2004; Quah et al., 2018; Robinson et al., 2001; Saleki-Haselghoubi et al., 2016; Wang et al., 1996a). In natural world, multibubble interaction is more commonly seen in the above mentioned applications. If there exists another bubble nearby, one bubble alters the ambient pressure field for the other bubble, which can significantly change the bubble dynamics and increase the bubble oscillation period (Bremond et al., 2006a; Cui et al., 2016a; Han et al., 2018; Ochiai and Ishimoto, 2017). On the contrary, the bubble period is shortened due to the existence of a free surface (Wang et al., 1996b). The combination of a free surface and an adjacent bubble may lead to more complex phenomena and this physical process is crucial to underwater explosions and seabed exploration. It is well known that the "whipping effect" is triggered when the oscillation frequency of a nearby underwater explosion bubble matches the resonance frequency of a naval ship (or a submarine) (Zhang and Zong, 2011; Zong et al., 2015). In the seabed exploration field, the bubble oscillation frequency or period is directly associated with the characteristics of the emitted sound wave, which is desired to contain more energy in the lowfrequency part (de Graaf et al., 2014; Zhang et al., 2018). The two-bubble system under consideration in this paper is the most extreme situation, i.e., the initial distance between two bubbles is smaller than 
the maximum equivalent bubble radius, leading to a strong interaction between them and the subsequent coalescence.

There are a number of studies on two-bubble dynamics near a free surface. In the numerical computation aspect, Robinson et al. (2001) studied the motion of single- and two-bubbles beneath a free surface using axisymmetric boundary integral method (BIM). Pearson et al. (2004) improved the model that can accurately capture the evolution of the free surface and the bubble motion. Liu et al. (2017) investigated the interaction between two identical oscillating bubbles and a free surface in a confined domain. Three-dimensional configurations were also considered in Han et al. (2014); Li and Ni (2016); Wang and Khoo (2004); Wang (2004). In these studies, the BIM based on the potential flow theory are commonly used for its high accuracy and efficiency. However, in the three-dimensional model, large deformations of the bubble interface and the free surface are somewhat problematic, which may lead to mesh distortion. Besides, topological transition included in bubble coalescence process is difficult to handle. To the authors' knowledge, there is no report on modelling of two-bubble coalescence beneath a free surface in the literature. This paper aims to establish a 3D model to overcome the above difficulties and to study the two-bubble coalescence beneath a free surface.

In the experimental aspect, multibubble interaction in a free field has been widely investigated (Bremond et al., 2006a; Bremond et al., 2006b; Chew et al., 2011; Fong et al., 2009) and the interaction between two bubbles and a rigid boundary has also been studied (Chew et al., 2013). It was found that multibubble dynamics were affected by many parameters including inter-bubble distance, bubbleboundary distance, size ratio, and phase difference. Ji et al. (2017) studied the effect of bubble-bubble distance and the water depth on the overall bubble behaviors. However, little attention has been paid on the coalesced bubble dynamics near a free surface.

In this study, a comprehensive numerical simulation complemented by physical experiments has been conducted to investigate the strong interaction between two oscillating bubbles and a free surface with particular focus on the dynamics of the coalesced bubble. Firstly, following the experimental method proposed by Turangan et al. (2006), the underwater discharge with a much higher voltage ( $580 \mathrm{~V}$ ) is used to generate oscillating bubbles. The maximum radius of a bubble reaches $\sim 15 \mathrm{~mm}$ with the oscillation bubble period $\sim 3 \mathrm{~ms}$. Two bubbles with similar size are generated simultaneously using the present experimental setup. High-speed photography technique is adopted to capture the bubble dynamic behaviors and free surface evolutions. The coalescence of two bubbles in different 
configurations beneath a free surface has been examined in detail. Secondly, both axisymmetric and three-dimensional boundary integral models are used to simulate the pre-coalescence and postcoalescence of two bubbles. Compared with previous investigations, one of the highlights in the present numerical study is that a higher resolution mesh is used and the latest numerical techniques including 3D topology treatment for bubble coalescence (Han et al., 2016), a weighted moving least square method for surface interpolation (Wang et al., 2015a; Wang and Manmi, 2014) and the Density Potential Method for mesh density control (Zhang and Liu, 2015) are implemented in the present numerical simulations. The present three-dimensional simulations have shown a good agreement with the axisymmetric model, indicating the high accuracy of the 3D model. More importantly, the essential physical phenomena in five representative experiments are well reproduced by the 3D modelling demonstrating the numerical models as effective tools to shed more light on the coalesced bubble dynamics, especially the collapse and jetting behaviors and the underlying mechanisms.

This paper is organized as follows. The experimental set-up is briefly introduced in Sec. 2. In Sec. 3, the strong interaction between two bubbles and a free surface and the coalescence of two bubbles are modelled using the boundary integral method, and mesh refinement techniques are also given. This is followed by Sec. 4 where five experiments are presented, in which two bubbles are arranged both in axisymmetric and three-dimensional configurations, and the main features of the bubble dynamics and free surface evolution are well reproduced by the present numerical model. In addition, pressure fields are calculated in three-dimensional cases for detailed analyses. In Sec. 5, the effects of dimensionless parameters on bubble collapse pattern and free-surface evolution are further discussed. Finally, the key conclusions are presented in Sec. 6.

\section{EXPERIMENTAL SETUP}

The experimental setup to investigate the interaction between two bubbles and a free surface is similar to that described in the work by Turangan et al. (2006) and sketched in Fig. 1. Oscillating bubbles are induced by electric discharge from a short circuit in water. Compared with the work by Turangan et al. (2006), a relatively high discharge voltage (580 V) is used in our experiments aimed at enlarging the bubble size and period. The maximum radius of an oscillating bubble generated in the present experiments is more than $10 \mathrm{~mm}$ making it possible to capture more physical details. It is noted that more than 150 consecutive frames in high-speed recordings are obtained during the first cycle of 
the coalesced bubble in the experiments with the spatial resolution being $0.23 \mathrm{~mm}$ per pixel.

In the electric circuit, switches $\mathrm{K} 1$ and $\mathrm{K} 2$ are responsible for charging and discharging, respectively. Firstly, switch K1 is turned on to charge a capacitor until an expected voltmeter reading is reached. Then the camera and switch K2 are triggered simultaneously by a control platform. The diameter of copper wires used as electrodes is $0.2 \mathrm{~mm}$, thus having little effect on bubble dynamics due to its much smaller diameter than that of an oscillating bubble. The cross point of two copper wires is referred to as the initial bubble center. Upon discharge, an oscillating bubble is generated by Joule heating at the cross point of the electrodes. Besides, series connection is assured to generate similarsized bubbles simultaneously and two cross points at predefined locations are the initial centers of the two in-phase bubbles, respectively. The initial distance between two predefined locations is set smaller than $15 \mathrm{~mm}$ to ensure the occurrence of the coalescence. In the experiment, a cubic container with dimensions of $500 \times 500 \times 500 \mathrm{~mm}^{3}$ is filled with water to a constant level of over $400 \mathrm{~mm}$. The dynamics of oscillating bubbles are recorded by a Phantom V711 high-speed camera which works at 52000 frames per second with an exposure time of $10 \mu \mathrm{s}$. Illumination is provided by a continuous light source from the back.

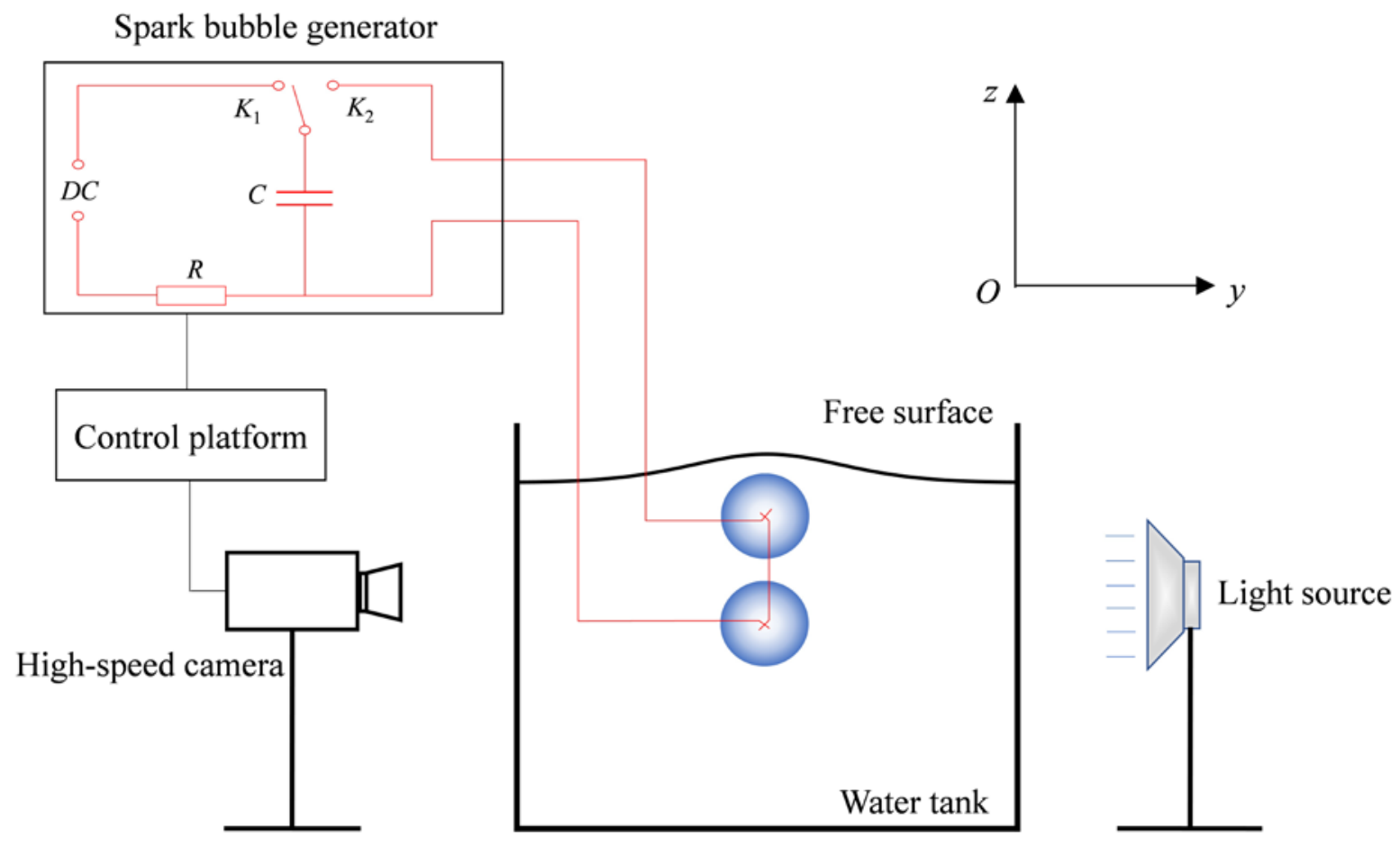

Fig. 1 Sketch of the experimental setup. 


\section{THEORETICAL AND NUMERICAL MODEL}

\subsection{Boundary Integral Method}

Axisymmetric and three-dimensional models based on the boundary integral method are both established to simulate the bubble dynamic behaviors and the evolution of the free surface. For two bubbles in a horizontal configuration, the left bubble is bubble 1 and the right one is bubble 2; otherwise, the upper bubble is bubble 1 and the lower one is bubble 2. A cylindrical coordinate $O-r \theta z$ and a Cartesian coordinate system $O-x y z$ are chosen for the axisymmetric model and the 3D model, respectively (Fig. 2). The origin $O$ is located at the initial center of bubble 1 with the $z$ axis pointing in the opposite direction of gravity. The maximum equivalent radii of the two bubbles are determined by their maximum bubble volumes, respectively, i.e., $R_{\max }=\left[3 V_{\max } /(4 \pi)\right]^{1 / 3}$. The distance between the initial center of bubble 1 and the free surface is denoted by $d_{b f}$ and the inter-bubble distance at the initial moment is denoted by $d_{b b}$.
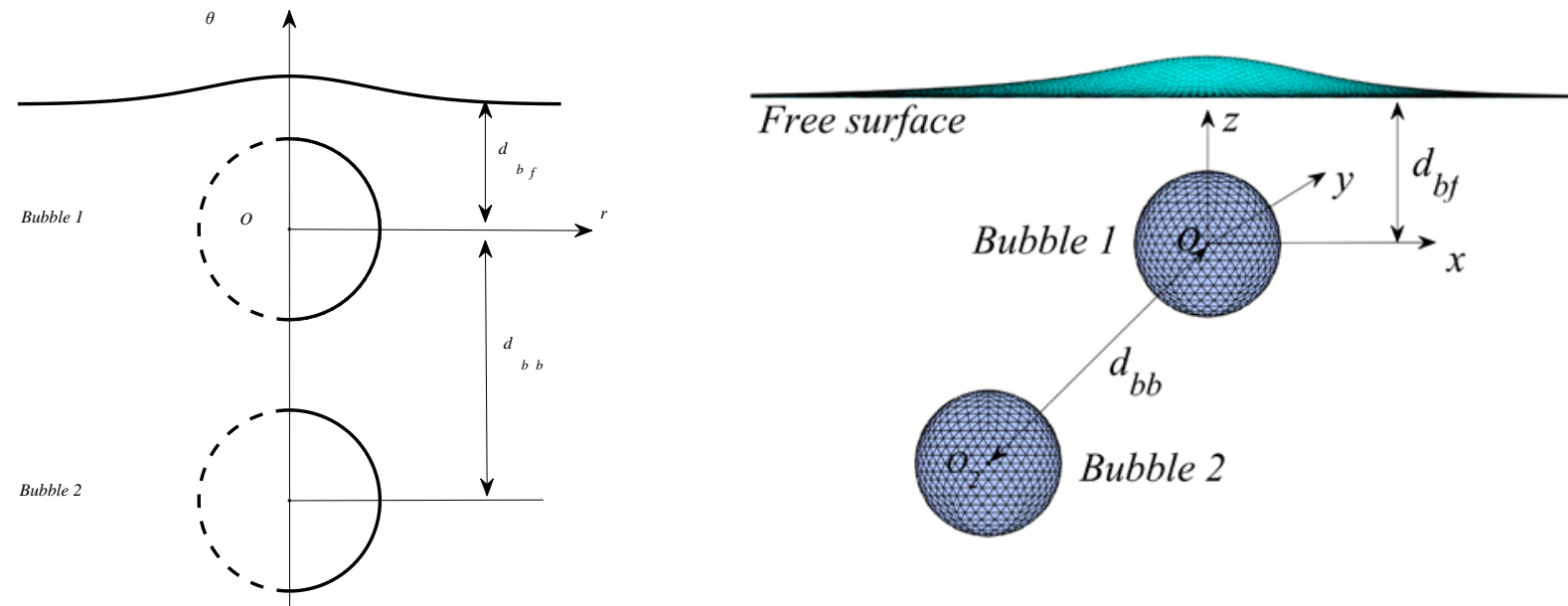

Fig. 2 Schematic view of the coordinate systems used in the (a) axisymmetric and (b) 3D models.

In the framework of the boundary integral method (Blake and Gibson, 1987; Han et al., 2016; Han et al., 2018; Pearson et al., 2004; Wang et al., 1996b; Zhang et al., 2015b; Zhang and Liu, 2015), the assumption that the fluid is inviscid and incompressible and the flow is irrotational in the timevarying domain is used. The velocity potential $\varphi$ in the fluid domain $\Omega$ is governed by Laplace's equation which can be transformed according to Green’s theorem:

$$
c(\boldsymbol{r}) \cdot \varphi(\boldsymbol{r})=\iint_{S_{b}+S_{f}}\left(G \cdot \frac{\partial \varphi(\boldsymbol{q})}{\partial n}-\varphi(\boldsymbol{q}) \frac{\partial G}{\partial n}\right) d S(\boldsymbol{q}),
$$


where $\boldsymbol{r}$ is the control point and $\boldsymbol{q}$ is the source point; $\boldsymbol{c}$ is the solid angle under which the fluid domain $\Omega$ is viewed from the control point $r ; \partial / \partial n$ is the normal outward derivative from the boundary $S$; the Green's function is $G=1 /|\boldsymbol{r}-\boldsymbol{q}|$.

Ignoring the surface tension and the heat transfer across the bubble surface, the internal pressure of the bubble $P_{b}$ is assumed to be related to the bubble initial state and its volume (Best and Kucera, 1992; Klaseboer et al., 2005a):

$$
P_{b i}=P_{0 i}\left(\frac{V_{0 i}}{V_{i}}\right)^{k}, \quad i=1,2,
$$

where $P_{0 i}$ and $V_{0 i}$ are the initial pressure and initial volume of the two bubbles, respectively; $V_{i}$ is the transient volume of the two bubbles; $k$ is the ratio of the specific heat for the gas and $k=1.25$ in this study.

The dynamic boundary conditions on the bubble surface and free surface yield:

$$
\begin{gathered}
\frac{\mathrm{d} \varphi}{\mathrm{d} t}=\frac{|\nabla \varphi|^{2}}{2}+\frac{P_{\infty}}{\rho}-\frac{P_{b}}{\rho}-g z, \\
\frac{\mathrm{d} \varphi}{\mathrm{d} t}=\frac{|\nabla \varphi|^{2}}{2}-g\left(z-d_{b f}\right),
\end{gathered}
$$

where $P_{\infty}$ is the hydrostatic pressure at the level of the initial center of bubble $1, \rho$ is the liquid density.

The kinematic boundary condition governing the motion of the bubble and the free surface is:

$$
\frac{\mathrm{d} \boldsymbol{r}}{\mathrm{d} t}=\nabla \varphi
$$

If two bubbles are initiated close to each other, coalescence may occur in the expansion phase. An important foundation of the present numerical model is the theoretical analysis by Bremond et al. (2006a) that the film thinning in the coalescence process of two expanding bubble is inertia dominated. Thus, the BIM is used to simulate the subsequent motion of the coalesced bubble beneath the free surface. In the calculation, the difference in the total bubble volume before and after coalescence is negligibly small and the heat transfer from the internal gases to the external liquid is also ignored within this short time. It is also assumed that the internal gases rapidly reach equilibrium after coalescence. Therefore, the pressure inside the coalesced bubble can be calculated using Equation (2). The initial pressure $P_{c 0}$ and volume $V_{c 0}$ of the coalesced bubble are given as below (Han et al., 2016; Rungsiyaphornrat et al., 2003): 


$$
\begin{gathered}
P_{c 0}=\left(P_{b 1} V_{1}+P_{b 2} V_{2}\right) /\left(V_{1}+V_{2}\right), \\
V_{c 0}=V_{1}+V_{2} .
\end{gathered}
$$

The axisymmetric calculations are carried out using both axisymmetric model and 3D model developed in the present study. In axisymmetric modelling, a downward jet is formed inside the bubble beneath a free surface and the topology treatment of the bubble surface at the impact moment is similar to the work by Wang et al. (1996a). The motion of the toroidal bubble is simulated by introducing a vortex ring with a strength equaling to the difference in $\varphi$ between the north and south poles of the bubble at the impact moment. An improved vortex ring model (Li et al., 2017; Zhang and Liu, 2015) is adopted in the present study. It is worth noting that the bubble dynamic behaviors both before and after the jet impact are simulated using the axisymmetric model. However, the calculation using 3D model for general three dimensional cases stops at the moment just before the jet impact due to the complexity associated with the topological transition of the 3D flow domain, and the physical and numerical instabilities in the toroidal bubble phase (Liu et al., 2016).

In the numerical simulations, quantities in dimensionless form are used. The maximum radius of bubble $1 R_{\max 1}$ is chosen as the length scale, the liquid density $\rho$ as the density scale and the ambient pressure at the initial centre of bubble 1 in the liquid $P_{\infty}$ as the pressure scale, respectively. Thus, $R_{\max 1}\left(\rho / P_{\infty}\right)^{0.5},\left(P_{\infty} / \rho\right)^{0.5}$ and $R_{\max 1}\left(P_{\infty} / \rho\right)^{0.5}$ are used as the characteristic time, velocity and velocity potential, respectively. At the initial moment, the distance between the center of bubble 1 and the free surface $\left(d_{b f}\right)$ and the inter-bubble distance $\left(d_{b b}\right)$ in dimensionless forms are denoted by $\gamma_{b f}$ (defined as the distance parameter) and $\gamma_{b b}$, respectively, together with the bubble size ratio $\alpha$, given as below:

$$
\gamma_{b f}=d_{b f} / R_{\max 1}, \gamma_{b b}=d_{b b} / R_{\max 1}, \alpha=R_{\max 2} / R_{\max 1} .
$$

The angle between the line connecting the initial bubble centers and the positive $x$-axis is defined as angle parameter $\beta$ to express the configuration, which is in the range of $[0, \pi / 2]$.

The dimensionless form of Equations (2)-(4) can be expressed as:

$$
\begin{aligned}
P_{b i}^{*} & =\varepsilon_{i}\left(\frac{V_{0 i}^{*}}{V_{i}^{*}}\right)^{k}, \quad i=1,2, \\
\frac{\mathrm{d} \varphi^{*}}{\mathrm{~d} t^{*}} & =\frac{\left|\nabla \varphi^{*}\right|^{2}}{2}+1-P_{b}^{*}-\delta^{2} z,
\end{aligned}
$$




$$
\frac{\mathrm{d} \varphi^{*}}{\mathrm{~d} t^{*}}=\frac{\left|\nabla \varphi^{*}\right|^{2}}{2}-\delta^{2}\left(z-\gamma_{b f}\right),
$$

where $\varepsilon=P_{0} / P_{\infty}$ is the dimensionless gas pressure of the initial bubble, and $\delta=\sqrt{\rho g R_{\max 1} / P_{\infty}}$ is the buoyancy parameter.

\subsection{Mesh topology treatment for bubble coalescence}

In the present BIM simulations, the flow boundaries in the time domain including the bubble surfaces and the free surface are tracked using a second-order Runge-Kutta scheme. If the inter-bubble distance is small, the surfaces of two bubbles would approach and flatten each other during the expansion phase, generating a thin liquid film between the two bubbles. Due to instabilities or other factors, the very thin liquid film would rupture and the coalescence of two bubbles occurs. However, this complex physical process is beyond the capability of BIM. Since BIM doesn't support a natural “merging” or split of bubbles, some artificial treatment of the topology should be made, as previously done in some literatures (Best, 1993; Lee et al., 2007; Rungsiyaphornrat et al., 2003; Wang et al., 1996a; Wang et al., 2015b; Zhang et al., 2015b; Zhang and Liu, 2015; Zhang et al., 2001). Following the work by Rungsiyaphornrat et al. (2003), a mesh topology treatment for bubble coalescence is performed if a simplified coalescence criterion is satisfied. Here we take the axisymmetric model to explain the procedure of "numerical coalescence" and the schematic of the topology treatment is given in Fig. 3. First, the coalescence treatment is triggered when the smallest distance between the two bubble surfaces is less than a critical value $s_{c}$. Second, the coalescence area (a liquid film to be removed later) is determined by the thickness of the water layer between the surfaces smaller than $2 s_{c} \sim 3 s_{c}$ (Han et al., 2016). Third, the nodes and elements on the coalescence area are removed and the remaining bubble surfaces are joined together by a 'stitch' line. In this step, the numbers of the nodes on the outermost rings of the coalescnece areas must be assured equal and new nodes are created at the midpoints between corresponding nodes, with the velocity potentials being the average values of those of correponding nodes. The difference in velocity potentials on the 'stitch' line is negligibly small and has insignificant effect on the subsequent motion of the coalesced bubble. The velocity potential elsewhere on the coalesced bubble surface keeps the same with that before coalescence. At last, a fivenode smooth scheme (Lundgren and Mansour, 1988) and node redistribution (Li et al., 2018b; Pearson et al., 2004; Zhang et al., 2015b) are implemented to control numerical instabilities of the coalesced 
bubble. For the axisymmetric model, the mesh topology treatment is easy to implement. However, the topology treatment involved in the 3D model is quite complex and the detailed procedures in numerical computation are given by Han et al. (2016).

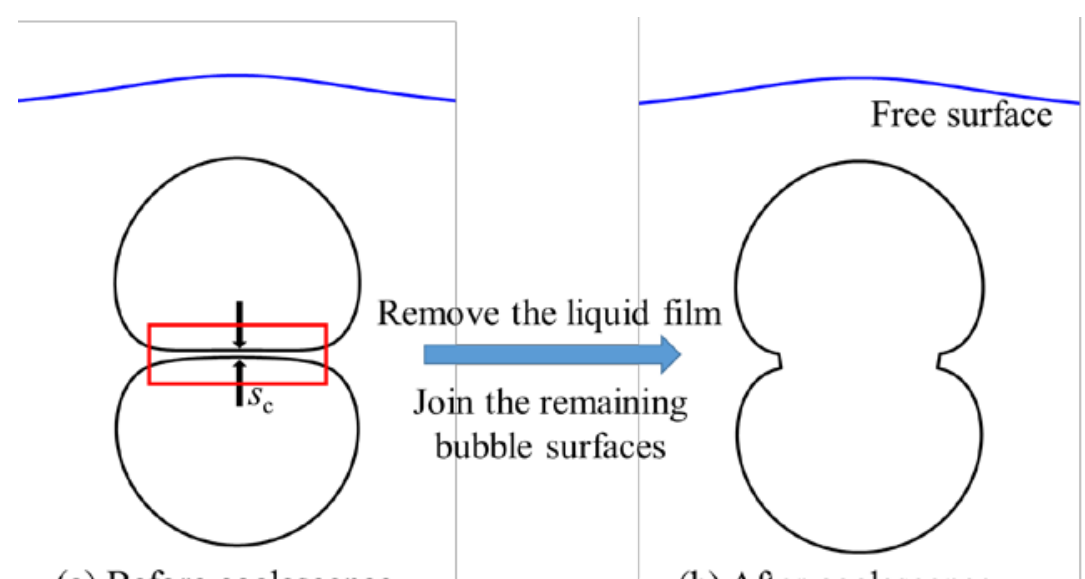

(a) Before coalescence

(b) After coalescence

Fig. 3 Sketch of the topology treatment during bubble coalescence.

To the best knowledge of the authors, there is little experimental report on the minimum thickness of a thin fluid layer before rupture. Here, the coalescence criterion is set as the dimensionless minimum thickness of the liquid film dropping below a constant value, namely $s_{c}$. Rungsiyaphornrat et al. (2003) set $s_{c}$ as 0.02 according to the experimental observations. In order to test the uncertainty of the physics of film rupture, sensitivity studies carried out by Han et al. (2016) measuring the influence of $s_{c}$ has demonstrated that the results are independent of $s_{C}$ in the range of $0.008<s_{C}<0.02$. Therefore, $s_{C}$ is set as 0.02 in the present study and the numerical results show favorable agreement with experiments.

Note that the velocity within the liquid film (before bubble coalescence) is quite small, it is reckoned that the above treatment of the bubble coalescence has little influence on the subsequent process of bubble motion (Han et al., 2016; Rungsiyaphornrat et al., 2003). It has been tested that the velocity variations at typical locations (e.g., bubble bottom, bubble top and the center of the free surface) caused by the "artificial treatment" are within $0.1 \%$. Additionally, overall quantitative agreements between the experiment and the numerical simulation can be found for two underwater explosion bubbles in a free field (Rungsiyaphornrat et al., 2003) and two cavitation bubbles below a rigid wall (Han et al., 2018). In the present study, five experiments of two-bubble coalescence beneath a free surface will be discussed and used to verify the numerical model. 


\subsection{Mesh refinement techniques}

Similar to previous studies in this field, numerical instabilities are found to occur during the evolution of the surfaces. Aimed at eliminating such instabilities, one of the highlights in the present study is that two techniques are adopted in the numerical simulations, namely, a smooth technique and the introduction of a mesh density controller. For the axisymmetric model, we use the five-point smooth scheme (Lundgren and Mansour, 1988) to eliminate the high-frequency saw-tooth instability. It is noted that the nodes on the bubble surface often overcrowd around the jet tip area, which reduces the time step and spatial resolution on other part of the bubble surface simultaneously. For this reason, the cubic spline interpolation is used for node redistribution (Zhang et al., 2015b).

Despite facing the same instability issue in 3D modeling, different solutions are used here. Following Zhang et al. (2001), a second order polynomial is applied to the bubble surface, and then the node positions and the velocity potentials on the nodes are interpolated using a weighted moving least square method (Han et al., 2016; Li et al., 2019). However, the node position is not updated by the material velocity, but the optimum shift velocity obtained by the Density Potential Method (Han et al., 2016; Zhang and Liu, 2015). A desired mesh density distribution can be obtained by adjusting the density potential function $\psi$. In the present study, different density potential functions are selected for bubbles and free surface. Meshes of high quality are often required for the jet zone of the bubbles, therefore the density potential function is selected according to the velocity potentials on the bubble surfaces. For the free surface, large deformations mainly occur around the central area of the whole free surface and the strong interaction between two bubbles and a free surface may lead to highly distorted meshes. Given this, the density potential function for the free surface is related to the distance parameter $\gamma_{b f}$, the positions of the nodes and the deformations of the meshes on the free surface. Density potential functions of the nodes on the bubble surface and free surface are set as follows:

$$
\begin{gathered}
\psi_{i}=\frac{\Lambda\left(\varphi_{i}\right) \cdot \sum_{j=1}^{N_{\text {ele }}} A_{i, j}}{N_{\text {ele }}}, \\
\psi_{i}=\frac{\Lambda\left(a z_{i}-b \sqrt{x_{i}^{2}+y_{i}^{2}}\right) \cdot \sum_{j=1}^{N_{\text {ele }}} A_{i, j}}{N_{\text {ele }}},
\end{gathered}
$$

where $N_{\text {ele }}$ is the total number of elements that are connected to node $i, A_{i, j}$ is the area of the $j^{\text {th }}$ element 
connected to node $i, \Lambda$ is a normalization operator, $a$ and $b$ are constants associated with $\gamma_{b f}$.

For further details about the Density Potential Method, see Zhang and Liu (2015) and Han et al. (2016). It is worth noting that the elastic mesh technique (Wang et al., 2003; Wang et al., 2015a) is also an alternative method to improve the mesh quality.

\section{RESULTS}

To understand the multibubble dynamics beneath a free surface, the present investigation starts from the experimental observations followed by comprehensive numerical simulations to further explore the underlying mechanisms of the coalesced bubble dynamics. In this section, five experiments are performed to validate the numerical model. Two bubbles with similar size are generated simultaneously beneath a free surface both in axisymmetric and three-dimensional configurations.

\subsection{Axisymmetric configuration}

In the experiments, two in-phase bubbles with similar size are generated beneath a free surface in an axisymmetric configuration. In the first experiment, the maximum equivalent radii of the two bubbles are $15.8 \mathrm{~mm}$ and $17.1 \mathrm{~mm}$, respectively. At the initial moment, the inter-bubble distance is $13.1 \mathrm{~mm}$ and the distance between the center of bubble 1 and the free surface is $23.6 \mathrm{~mm}$. Thus the dimensionless parameters are: $\gamma_{b f}=1.49, \gamma_{b b}=0.83, \alpha=1.08$.

The shapes of the two bubbles in the experiment at selected times are given in Fig. 4 (a). Two bubbles are seen expanding quickly after the inception (frame 1) and the liquid film between the interfaces becomes thinner and thinner as the two bubbles approaching each other (frame 2). Due to limitations of the present experimental setup, it is difficult to observe the process of the film rupture directly. Bremond et al. (2006a) predicted the bubble coalescence by a morphology change in interface shape, namely, the disappearance of the cusp between two bubbles (indicated by a red arrow in Fig. 3 (a)). The interface is smoothed by the surface tension once the liquid film ruptures which therefore reveals the coalescence of the bubbles (between frames 3 and 4). A circular "scar" on the bubble surface can be observed after the coalescence of the bubbles takes place (frame 4). The coalesced bubble attains the maximum volume at $1.87 \mathrm{~ms}$ and the top of the coalesced bubble is of a higher curvature, which

indicates a faster collapse speed subsequently. Besides, since the coalesced bubble is subject to the 
Bjerknes force from the free surface, the top of the coalesced bubble contracts faster than other parts of the bubble during the collapse phase and a wide downward jet with a flattened tip is formed afterwards (frames 5 and 6). Blake et al. (1987) and Wang et al. (1996b) provided explicit explanations to the downward-jet formation near a free surface, i.e., the fluid is drawn into the region between the bubble top and the free surface during the bubble collapse phase, resulting in the formation of a stagnation point and a high-pressure region along the $z$-axis. This localized high pressure region drives the bubble top downward and the water spike upward. The jet further develops and finally penetrates the bottom of the coalesced bubble (frame 7). Thereafter, the collapsing coalesced bubble transforms from singly-connected into doubly-connected forms. The toroidal bubble keeps collapsing and a protrusion forms at the bottom of the bubble afterwards. The bubble splits into two parts at the intersection between the protrusion and the main bubble (frame 8). It is also noticed that the circular "scar" on the bubble surface due to coalescence is gradually detaching from the bubble surface, as shown in frames 5-8, which is presented as the annular residual (indicated by red arrows in frame 6) near the coalescence position of the coalesced bubble.

The numerical results using axisymmetric model (red lines) and three-dimensional model at corresponding times are presented in Fig. 4 (b). Obviously, the results obtained by two models agree well with each other in this axisymmetric case, in terms of bubble surface and free surface evolutions. The small discrepancy is attributed to the mesh size/resolution difference between the two models. Because a simple numerical treatment is adopted to handle the bubble coalescence problem, the annular residual near the coalescence position in the experiment cannot be obtained. However, the main features of the coalesced bubble beneath a free surface in the experiment is well reproduced by the present numerical models. Due to the relatively large distance between bubbles and free surface, only a small bulge on the free surface is induced by the growth of the upper bubble. During the collapse phase of the coalesced bubble, the bulge on the free surface increases slowly and gradually becomes slimmer. 
(a)
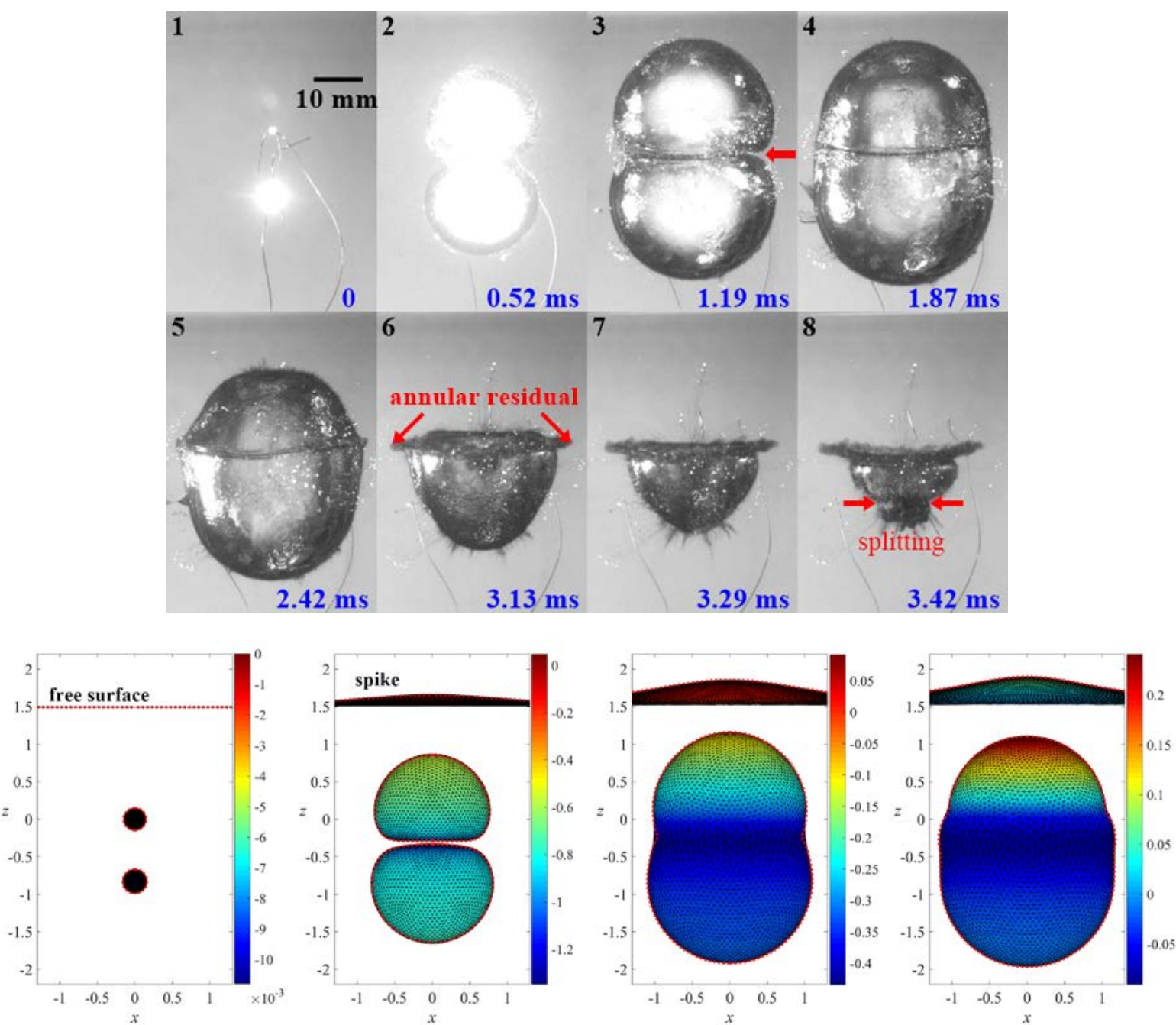

(b)
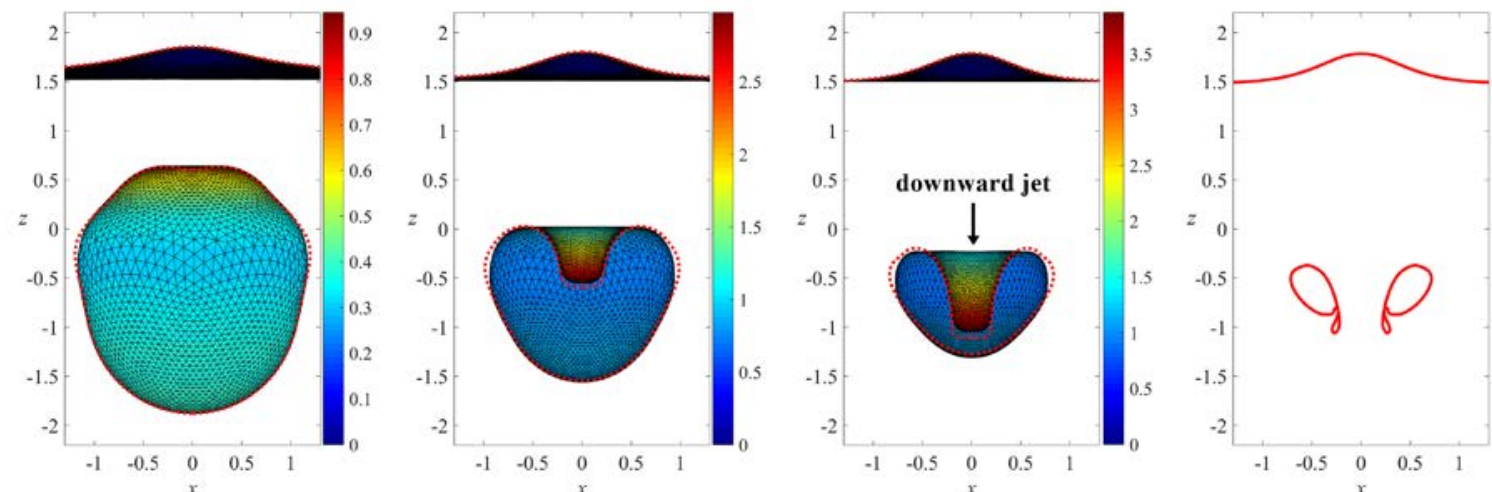

Fig. 4 Comparison of the dynamics of a coalesced bubble beneath the free surface between (a) experimental observations (with the corresponding time marked at the bottom right corner of each image) and (b) numerical results (the red lines represent the results obtained by the axisymmetric model, the contours represent the velocity potential). Experimental parameters are: $R_{\max 1}=15.8 \mathrm{~mm}$, $R_{\max 2}=17.1 \mathrm{~mm}, d_{b f}=23.6 \mathrm{~mm}$ and $d_{b b}=13.1 \mathrm{~mm}$. Dimensionless parameters in the numerical simulation are set according to the experiment: $\gamma_{b f}=1.49, \gamma_{b b}=0.83, \alpha=1.08, \varepsilon=100, R_{01}=0.1485$ and $R_{02}=0.1604$. The dimensionless times of the numerical results are $0,0.321,0.746,1.078,1.504$, $1.921,2.069$ and 2.151, respectively (the time scale is $1.596 \mathrm{~ms}$ ). 
In the second experiment, the maximum equivalent radii of the two bubbles are $16.2 \mathrm{~mm}$ and 15.6 mm, respectively. The distance between the initial center of bubble 1 and the free surface, and the initial inter-bubble distance are both $12 \mathrm{~mm}$, corresponding to the following dimensionless parameters: $\gamma_{b f}=0.74, \gamma_{b b}=0.74, \alpha=0.96$. Due to a much smaller $\gamma_{b f}$ in this case, the bubble-free-surface interaction is much stronger, as shown in Fig. 5 (a). More specifically, the two bubbles expand rapidly, become flattened at their adjacent surfaces, and deform the free surface significantly in the meantime (frames 1-2). Due to the closer proximity of the upper bubble to the free surface, the top of bubble 1 is entrained into the base of the raised free surface and the spike gains a relatively larger height compared with case 1 (frame 3). The coalescence of the two bubbles occurs around frame 3. Meanwhile, the free-surface spike elevates with the volume increase of the coalesced bubble. During the collapse phase of the coalesced bubble, a relatively thinner jet (compared with case 1) directed downwards is produced. It is noticed that the jet tip is unstable, which is companied with the splashing and splitting of tiny droplets. These droplets might shoot on the inner surface of the bubble, which is responsible for the rough bubble surface observed in frame 7. The liquid jet further develops and finally impacts the bottom of the coalesced bubble (frames 7-8). After the jet impact, the coalesced bubble evolves into a toroidal bubble and the downward protrusion is also quite unstable and is about to split from the toroidal bubble in frame 8. The free surface continues rising along the axis of symmetry and meanwhile shrinking in width at the base, producing a thin and sharp free-surface spike.

Numerical results using axisymmetric and three-dimensional models at corresponding times are given in Fig. 5 (b). Although the strong interaction between two bubbles and the free surface leads to a large deformation of the free surface, the overall evolutions of the coalesced bubble and the freesurface spike in the experiment are also well captured by our numerical models. A liquid jet with a flattened tip is seen formed inside the coalesced bubble. Though the splashing effect observed in the experiment is beyond the capability of the present numerical model, the jetting phenomenon and collapse pattern of the coalesced bubble are well captured. A slight difference in the height of the freesurface spike are observed between the two models, which may be attributed to the fact that the mesh density in 3D simulation is lower than that in the axisymmetric model. However, both models are of high accuracy to capture the main features of the coalesced bubble and the free surface motion. The axisymmetric model also reproduces the splitting of the toroidal bubble, as shown in the last frame of Fig. 5 (b). 
(a)

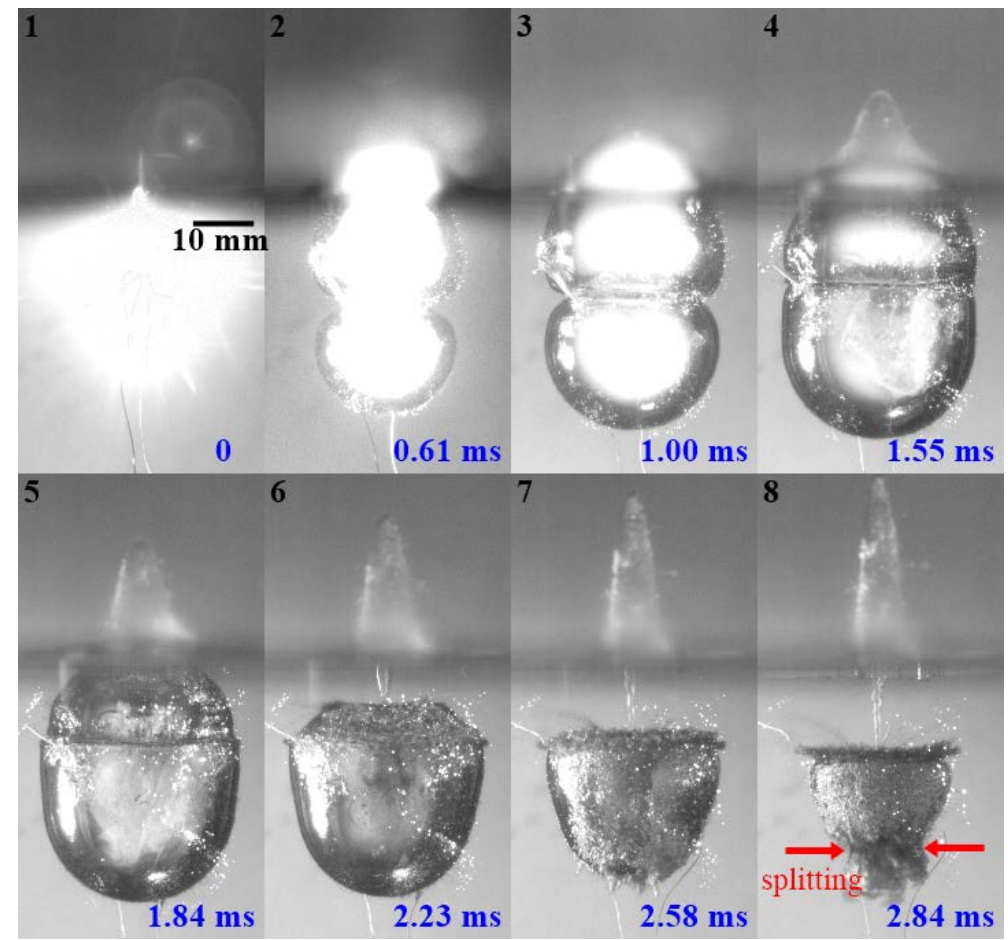

(b)
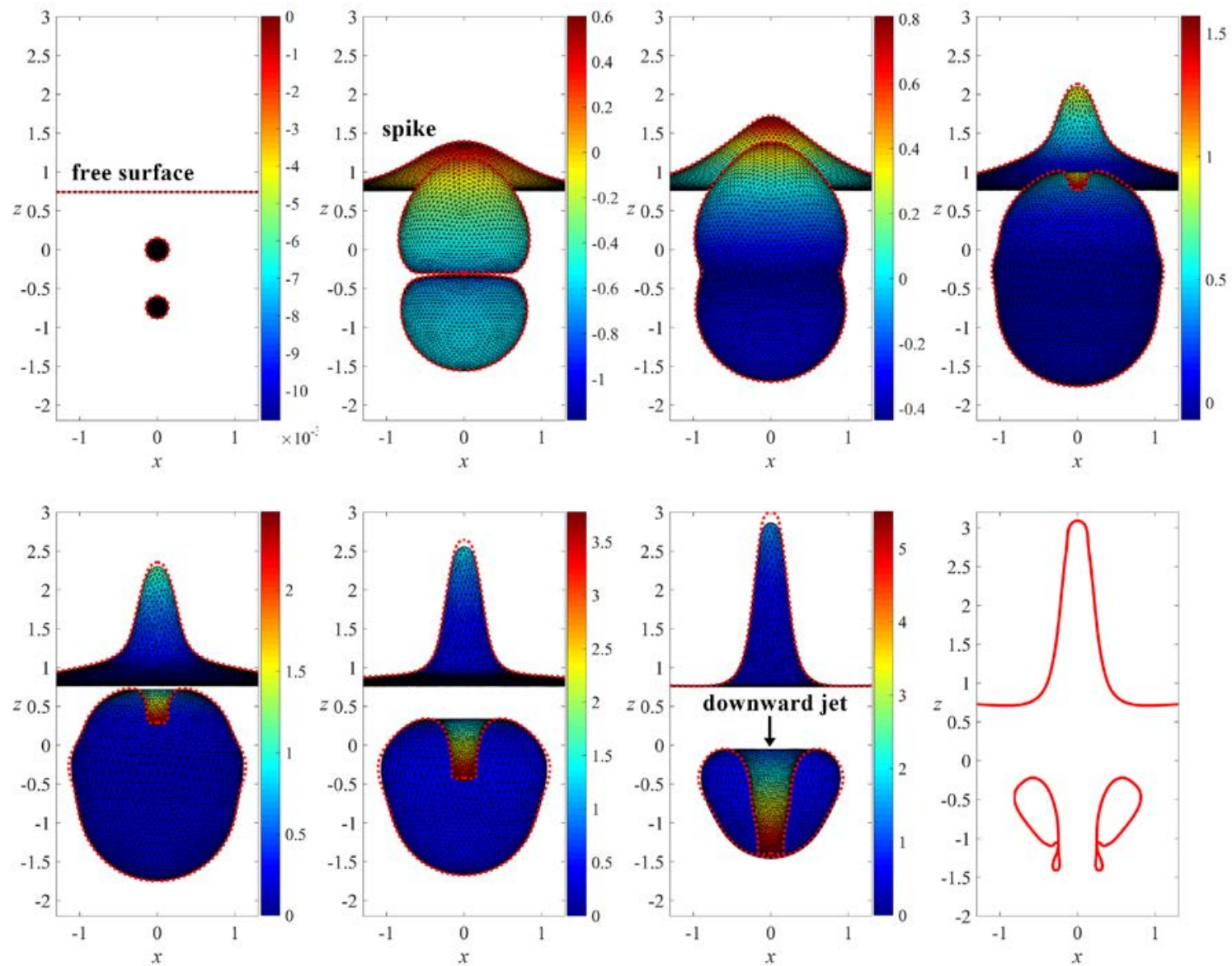

Fig. 5 Comparison of the dynamics of a coalesced bubble beneath the free surface between (a) experimental observations (with the corresponding time marked at the bottom right corner of each image) and (b) numerical results. Experimental parameters are: $R_{\max 1}=16.2 \mathrm{~mm}, R_{\max 2}=15.6 \mathrm{~mm}$, 
$d_{b f}=12 \mathrm{~mm}$ and $d_{b b}=12 \mathrm{~mm}$. Dimensionless parameters in the numerical simulation are: $\gamma_{b f}=0.74$, $\gamma_{b b}=0.74, \alpha=0.96, \varepsilon=100, R_{01}=0.1485$ and $R_{02}=0.1431$. The dimensionless times of the numerical results are $0,0.371,0.609,0.947,1.124,1.363,1.672$ and 1.795 , respectively (the time scale is 1.636 $\mathrm{ms})$.

\subsection{Three-dimensional configuration}

In this section, two bubbles with similar size are generated simultaneously beneath the free surface in three-dimensional configurations. Bubbles are arranged in a horizontal line or in an oblique line and some interesting phenomena are observed. Three-dimensional numerical simulations are presented in comparisons with the experiments followed by detailed analyses of bubble dynamics based on pressure fields calculated.

In the third experiment (case 3), the maximum equivalent radii of the two bubbles are $16.5 \mathrm{~mm}$ and $17.3 \mathrm{~mm}$, respectively. At the initial moment, the inter-bubble distance is $10.4 \mathrm{~mm}$ and the distance between the center of bubble 1 and the free surface is $24.9 \mathrm{~mm}$ resulting in the following dimensionless parameters: $\gamma_{b f}=1.51, \gamma_{b b}=0.63, \alpha=1.05$. Experimental observations and three-dimensional numerical simulations are compared in Fig. 6. The significant expansion of the two bubbles is seen inducing a wide bulge on the free surface and the two bubbles are approximately semi-spherical with flattened interfaces (frames 1-2). Following the rupture of the thin liquid film between two bubbles, the coalesced bubble continues to expand and attains the maximum volume in frame 4; meanwhile the wide bulge on the free surface further rises. The vertical dark line in frames 6-8 (indicated by a red arrow) is the annular residual mentioned in Sec. 4.1. The liquid jets cannot be clearly observed in the experiment but can be inferred by the numerical simulation (discussed later). In addition, the coalesced bubble is observed migrating away from the free surface during the collapse phase. 
(a)

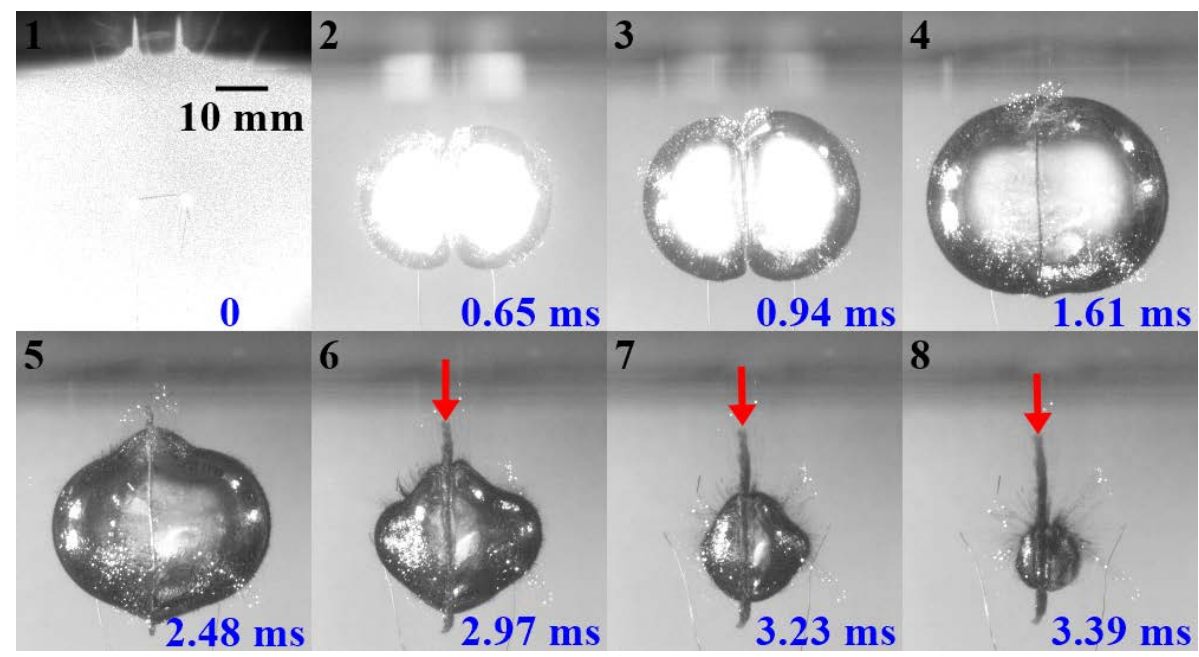

(b)

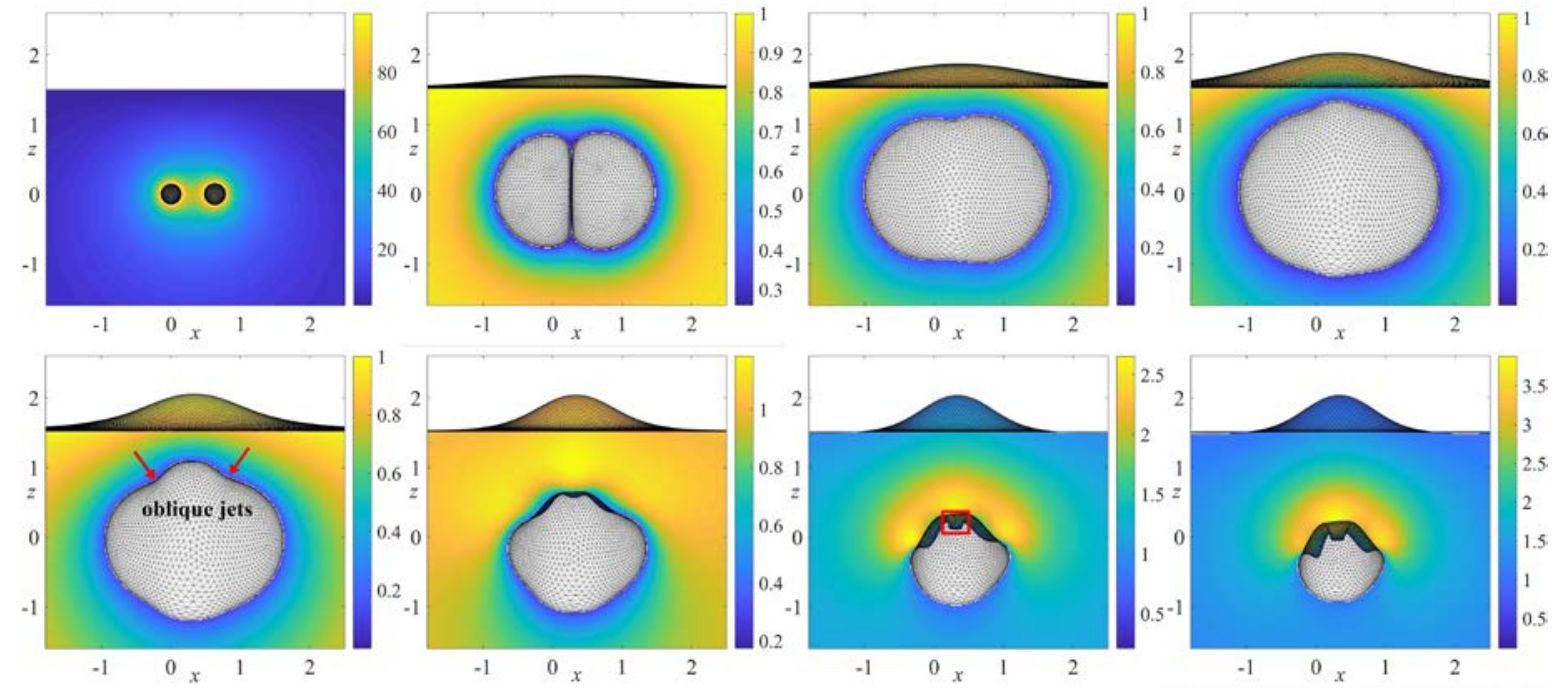

Fig. 6 Comparison of the dynamics of a coalesced bubble beneath the free surface between (a) experimental observations (with the corresponding time marked at the bottom right corner of each image) and (b) numerical results. Experimental parameters are: $R_{\max 1}=16.5 \mathrm{~mm}, R_{\max 2}=17.3 \mathrm{~mm}$, $d_{b f}=24.9 \mathrm{~mm}$ and $d_{b b}=10.4 \mathrm{~mm}$. Dimensionless parameters in the numerical simulation are: $\gamma_{b f}=$ 1.51, $\gamma_{b b}=0.63, \alpha=1.05, \varepsilon=100, R_{01}=0.1485$ and $R_{02}=0.1559$. The dimensionless times of the numerical results are $0,0.311,0.565,0.967,1.488,1.781,1.937$ and 1.980 , respectively (the time scale is $1.668 \mathrm{~ms})$.

Numerical results present the jet formation and development inside the coalesced bubble and also the evolution of the pressure fields surrounding the bubbles, as shown in Fig. 6 (b). After the inception, the two bubbles expand fast due to the high internal pressure and the overexpansion is restrained by the surrounding pressure. During the expansion phase, the pressure field surrounding the bubble is relatively uniform (frames 1-4). However, due to the existence of the free surface, the pressure gradient between the free surface and the bubble top is the largest, which indicates a faster contraction of the 
upper part of the coalesced bubble. If there is no free surface, two jets originate on the left and right surfaces of the coalesced bubble directed horizontally towards each other (Cui et al., 2016a; Han et al., 2016); however, the existence of a free surface affects the jet directions greatly. Due to the mutual attraction of the two oscillating bubbles and the free-surface Bjerknes force, the upper left and upper right surfaces of the coalesced bubble contract earlier and initially form two oblique jets (indicated by red arrows in frame 5). Hence, the surrounding fluid is quickly drawn into the regions above the coalesced bubble and a large high-pressure region is gradually formed (frame 6). A downward jet with a flattened tip (circled by a red rectangle in frame 7) then originates on the top of the coalesced bubble. Three localized high-pressure regions are observed in frame 7, which promote the development of the three jets. Due to the Bjerknes force and the effect of the downward jet, the two oblique jets gradually deviate from the original directions but still keep squeezing the downward jet together. In the later stage of the collapse phase, the three jets squeeze each other as demonstrated in the numerical results, indicating that the three jets may collide inside the coalesced bubble in the experiment. In fact, the development of the three jets makes the mesh highly distorted and irregular, so the calculation stop before 3.39ms, which causes the slight discrepancies in bubble shapes in the final collapse phase between the experiment and the simulation. The formation of one oblique jet during bubble collapse can be observed in other configurations (Brujan et al., 2018; Cui et al., 2016a; Li and Ni, 2016; Liu et al., 2016; Ochiai and Ishimoto, 2017; Rosselló et al., 2018; Tagawa and Peters, 2018), however, multiple oblique jets are rarely reported before.

In the fourth experiment (case 4), the maximum equivalent radii of the two bubbles are both about $16.6 \mathrm{~mm}$. The initial inter-bubble distance is $14.1 \mathrm{~mm}$ and the initial distance between the center of bubble 1 and the free surface is reduced to $12.1 \mathrm{~mm}$, corresponding to the following dimensionless parameters: $\gamma_{b f}=0.85, \gamma_{b b}=0.73, \alpha=1$. Comparison between the experiment and the numerical results is illustrated in Fig. 7. It can be observed that the two bubbles are entrained into the base of the free surface spike in the expansion phase. Subsequently, the coalesced bubble is elongated along the z-axis (frames 1-4). One of the interesting features can be seen is that the upper part of the coalesced bubble collapses faster and two oblique jets are initially formed due to the mutual interaction of the two bubbles and the effect of the free surface (frames 5-6). Annular residual is also observed in this case (see the vertical dark line in frames 6-8). Although the annular residual makes it difficult to observe the motion of the bubble top, the numerical prediction reveals that the top of the coalesced bubble 
collapses fast under the strong effect of the free surface and is most likely to form a downward liquid jet. In this case, a wide bulge on the free surface appears with the expansion of the coalesced bubble and the evolution of the free-surface spike is clearly evident. The free-surface spike keeps rising and narrowing at the base and a flattened tip of the spike is observed in the front view.

(a)

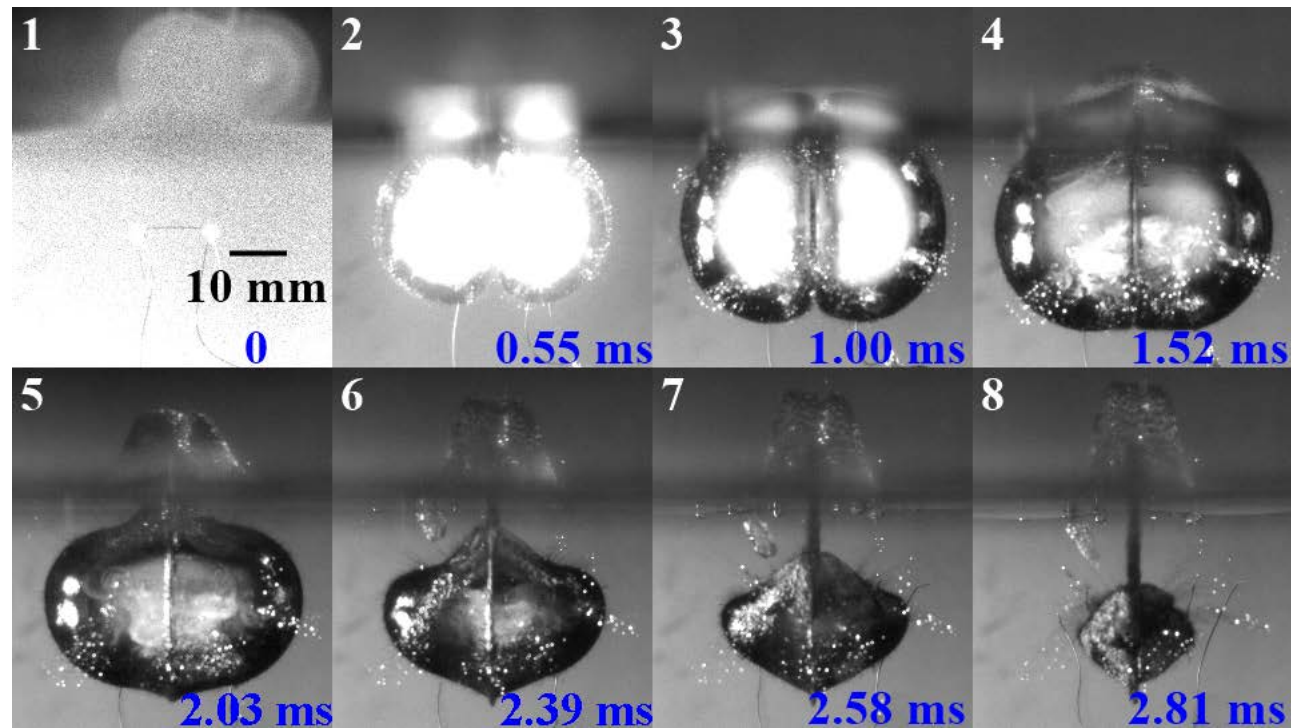

(b)
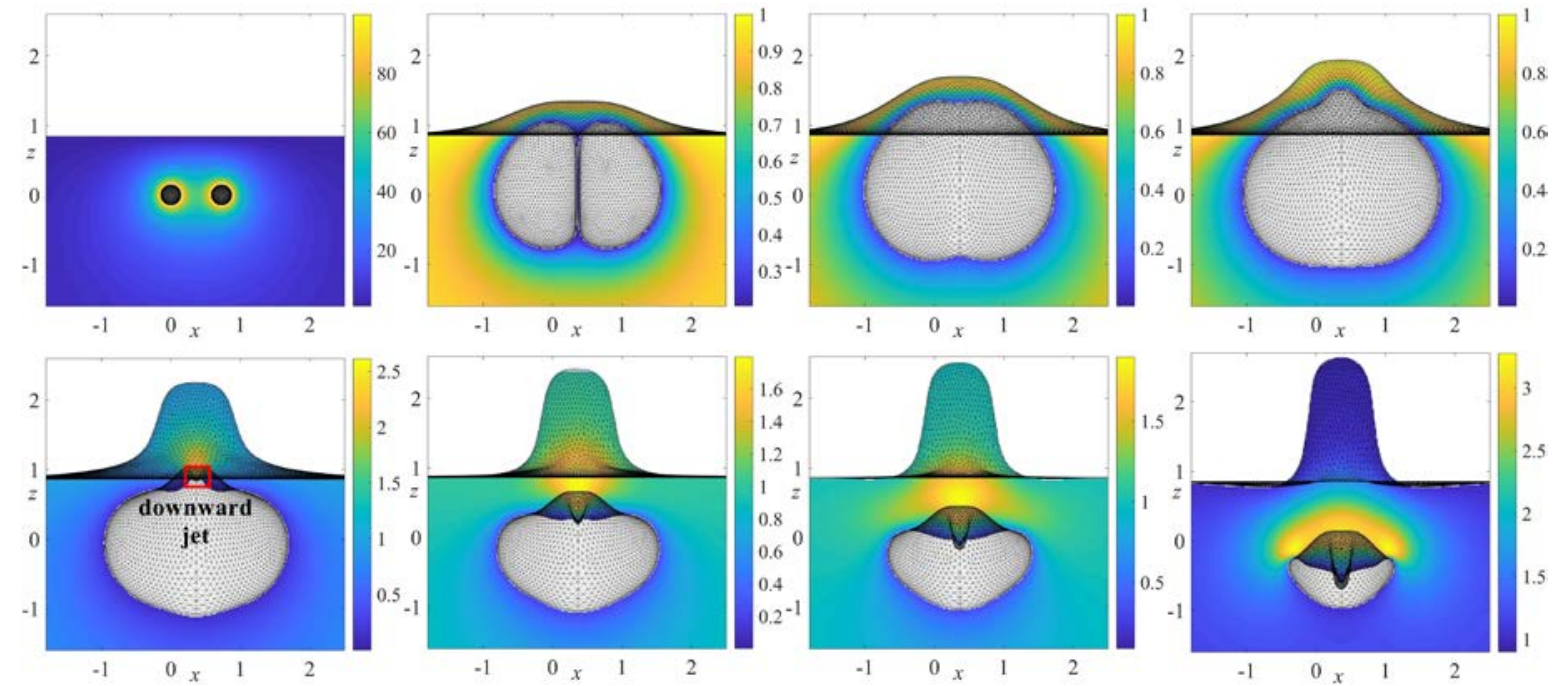

Fig. 7 Comparison of the dynamics of a coalesced bubble beneath the free surface between (a) experimental observations (with the corresponding time marked at the bottom right corner of each image) and (b) numerical results. Experimental parameters are: $R_{\max 1}=16.6 \mathrm{~mm}, R_{\max 2}=16.6 \mathrm{~mm}$, $d_{b f}=12.1 \mathrm{~mm}$ and $d_{b b}=14.1 \mathrm{~mm}$. Dimensionless parameters in the numerical simulation are: $\gamma_{b f}=$ 0.85, $\gamma_{b b}=0.73, \alpha=1, \varepsilon=100, R_{01}=0.1485$ and $R_{02}=0.1485$. The dimensionless times of the numerical results are $0,0.326,0.596,0.846,1.211,1.422,1.538$ and 1.701 , respectively (the time scale is $1.678 \mathrm{~ms}$ ). 
Numerical simulation reveals the detailed jet formation and development, as given in Fig. 7 (b). In the expansion phase, it is clearly observed that the top surface of the coalesced bubble is entrained within the base of the free-surface spike and a small protrusion with a high curvature appears at the bubble top (frames 1-4), which indicates that the top of the coalesced bubble will contract faster subsequently. The pressure distribution in the flow field also predicts the faster collapse of the upper part of the coalesced bubble, i.e., the pressure gradient above the bubble top is the largest in the flow. In the collapse phase, two oblique jets originate on the upper left and right surfaces and an initial formation of a downward jet (circled by a red rectangle in frame 5) is also observed. Meanwhile, a high-pressure region is formed around the top of the coalesced bubble, which promotes the development of the downward jet and the free-surface spike. It is noted that the positions where the two oblique jets originate approach more towards the bubble top compared with that in case 3 . The downward jet develops fast due to the positive feedback effect of the high-pressure region (Koukouvinis et al., 2016; Li et al., 2018a) and is squeezed by the two oblique jets all along, thus obtaining a small width in the front view; while the two oblique jets are not clearly observed but the contraction of the upper left and right surfaces indicate the development of the two jets. The surrounding fluid is rapidly drawn into the regions between the coalesced bubble and the free surface as the jet develops and the high-pressure region gradually becomes larger (frames 6-8).

A side view of the bubble and free-surface profiles corresponding to frames 3-8 in Fig. 7 (b) is given to illustrate the evolution of the interesting jet, as shown in Fig. 8. From this view, the bubble and free-surface profiles are similar to those in single-bubble case (frames 1-2). The fast contraction of the bubble top draws the surrounding fluid into the region between the bubble and the free surface, and a stagnation point is formed along the $z$-axis at $y=0$, thus leading to the formation of a highpressure region (between frames 2 and 3). In frame 3, a downward jet with a flattened tip (circled by a red rectangle) is observed and a wide secondary jet due to the large high-pressure region is formed at the base of the downward jet. As the jet evolves, the high-pressure region becomes larger, which pushes the wide secondary jet (indicated by red arrows) to develop so fast that the first downward jet cannot be observed in frame 4. As the downward jet is squeezed by the two oblique jets, a sheet-like liquid jet is gradually formed with a large width in side view. Finally, there are two contact points at the jet impact moment (frames 3 and 4). The free-surface spike keeps rising due to the inertia and becomes thinner at the base. 

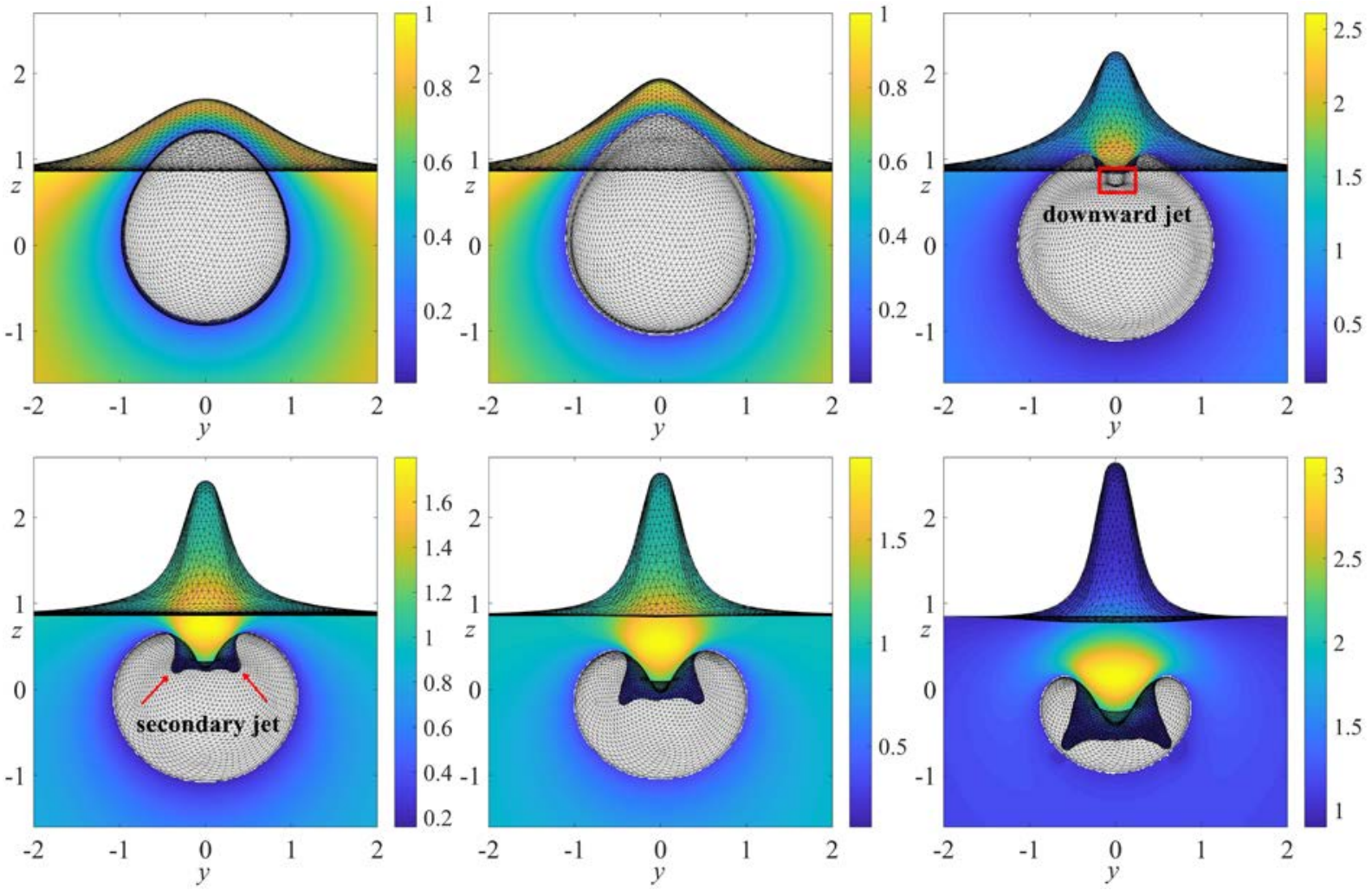

Fig. 8 Side view of the bubble and free-surface profiles corresponding to frames 3-8 in Fig. 7 (b). The dimensionless times are $0.596,0.846,1.211,1.422,1.538$ and 1.701 , respectively

The interaction between two bubbles in an oblique configuration near a free surface is also investigated. In the fifth experiment, both the maximum equivalent radii of the two bubbles are about $11 \mathrm{~mm}$. At the inception moment, the distance between the centers of the two bubbles is $7.0 \mathrm{~mm}$, with the vertical and horizontal components being $2.5 \mathrm{~mm}$ and $6.6 \mathrm{~mm}$, respectively, and the vertical distance between the center of bubble 1 and the free surface is $11 \mathrm{~mm}$. Thus the dimensionless parameters are: $h_{x}=0.60, h_{z}=0.23, \gamma_{b f}=1, \gamma_{b b}=0.64, \alpha=1$. The experimental and numerical results are compared in Fig. 9. In this case, the angle between the axis connecting initial bubble centers and the positive $x$-axis is about $22^{\circ}$ (i.e. $\beta=0.38$ ). In the experiment, the top of the upper bubble (bubble 1) is entrained within the free surface in the expansion phase (frame 2) and then a downward jet is formed inside the coalesced bubble due to the free-surface effect (frames 3 and 4 ). The jet is about to impact the bubble bottom in frame 5 . Though the liquid jet is not clearly observed in the experiment, the subsequent motion of the coalesced bubble indicates the formation of a downward jet. In this case, an oblique free-surface spike is observed and the spike keeps elevating in an oblique direction with its base narrowing. 
(a)

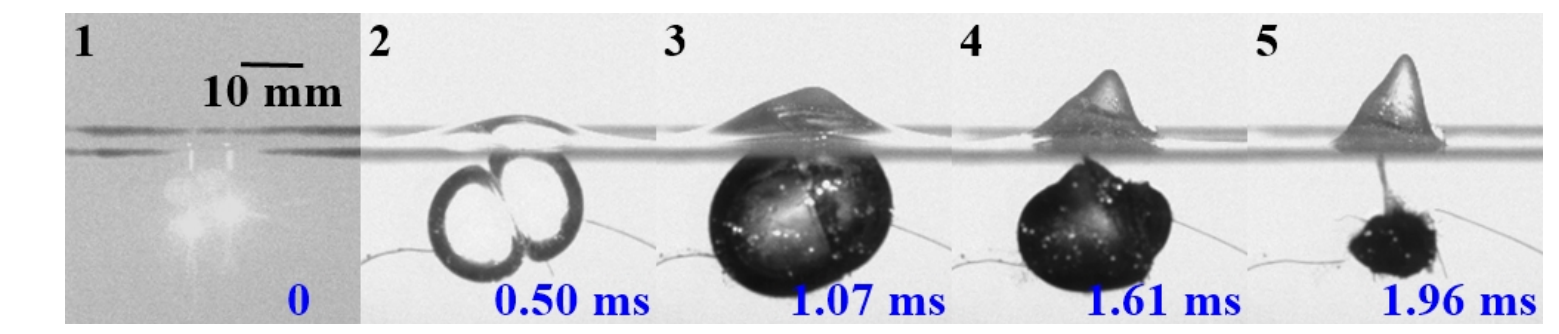

(b)

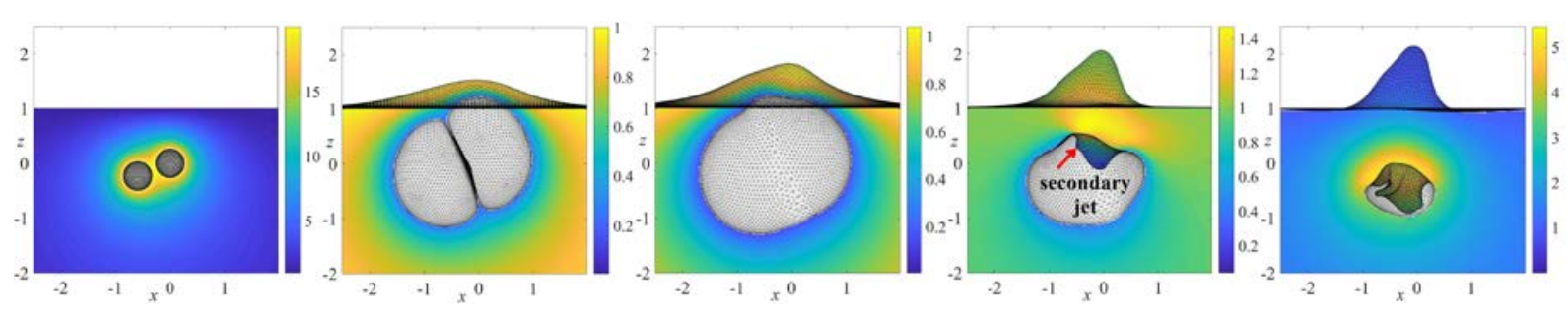

Fig. 9 Comparison of the dynamics of a coalesced bubble beneath the free surface between (a) experimental observations (with the corresponding time marked at the bottom right corner of each image) and (b) numerical results. Experimental parameters are: $R_{\max 1}=11 \mathrm{~mm}, R_{\max 2}=11 \mathrm{~mm}, d_{b f}=$ $11 \mathrm{~mm}$ and $d_{b b}=7 \mathrm{~mm}$. Dimensionless parameters in the numerical simulation are: $\gamma_{b f}=1.0, \gamma_{b b}=$ 0.64, $\alpha=1, \varepsilon=100, R_{01}=0.1485$ and $R_{02}=0.1485$. The dimensionless times of the numerical results are $0,0.449,0.960,1.446$ and 1.810 , respectively (the time scale is $1.113 \mathrm{~ms}$ ).

The numerical results at corresponding times are given in panel (b). In frame 2, coalescence of two bubbles is about to occur in the simulation and the top of bubble 1 is entrained within the free surface, leading to a slight elongation of bubble 1 along the $z$-axis. The coalesced bubble continues to expand and the free-surface spike elevates with a small horizontal deviation (frame 3). The top of the coalesced bubble contracts first due to its higher curvature and the free-surface Bjerknes force dominates the jet direction due to the close proximity of bubble 1 to the free surface. The localized high-pressure region near the bubble top promotes the development of the downward jet and the oblique free-surface spike (between frames 3 and 4). Afterwards, the pressure peak of the high-pressure region moves towards the bubble top as the downward jet develops. The migration of the pressure peak leads to the formation of a secondary jet which originates on the top of the coalesced bubble (indicated by a red arrow in frame 4). The downward jet and the secondary jet squeezes each other during the development process; thus, a bubble jet with several corners is observed when the downward jet finally impacts on the bubble bottom (frame 5). At that moment, a large high-pressure region is observed. The oblique spike in the experiment is also captured by the simulation. 


\section{DISCUSSIONS}

The interaction between two identical bubbles and a free surface is mainly affected by four parameters, i.e., the distance parameter $\gamma_{b f}$, the inter-bubble distance $\gamma_{b b}$, the angle parameter $\beta$ and the buoyancy parameter $\delta$. The present study focuses on the dynamics of a coalesced bubble and the $\gamma_{b b}$ ranges from 0.63 to 0.73 in the experiments, thus the effect of $\gamma_{b b}$ is not under consideration in this paper. More discussion on the effect of $\gamma_{b b}$ on bubble coalescence in a free field can be found in Han et al. (2016). In this section, a parametric study reveals the dependence of the coalesced bubble dynamics and free surface evolutions on $\gamma_{b f}$ and $\beta$. According to a set of practical data in airgun-bubble clusters (Cox et al., 2004), the other parameters are set as: $\alpha=1, \varepsilon=140$ and $\delta=0.22$. The buoyancy parameter is also similar to that of underwater explosion bubbles and the strength parameter does not strongly affect the bubble dynamic behaviors before jet impact moment, thus the results shown in this section also yield new insight into underwater explosions.

From the experiments described in Section 4, it is noted that $\gamma_{b f}$ and $\beta$ have significant effects on the bubble dynamics and free-surface motion. To investigate the effect of $\beta$, let us set the value of $\beta$ ranging from 0 to $\pi / 2$. On the axis connecting two initial bubble centers, the end of bubble 1 away from bubble 2 is defined as pole A (PA for short) and the end of bubble 2 is defined as pole B (PB for short). If two bubbles are initiated in an infinite field without gravity effects, two jets are formed at the two ends of the bubble (defined as $\mathrm{PA}^{\prime}$ and $\mathrm{PB}^{\prime}$ ) and they develop along the connecting line with opposite directions. In this case, $\mathrm{PA}^{\prime}$ and $\mathrm{PB}$ ' coincide with $\mathrm{PA}$ and $\mathrm{PB}$, respectively. However, the existences of a free surface and the buoyancy have effects on the positions where the two jets originate and redirect the two jets accordingly.

\subsection{Weak bubble-free-surface interaction with different $\beta$}

In the first series of simulations, similar dimensionless parameters with those in case 3 are selected, i.e., $\gamma_{b b}=0.63$ and $\gamma_{b f}=1.5$. Bubble and free surface profiles at the jet impact moment with different $\beta$ are given in Fig. 10, the two initial bubble centers and the connecting line are also plotted. The evolution of the coalesced bubble and the free surface with $\beta=0$ is similar to that in case 3 and three jets are observed to squeeze each other (see Fig. 6). When $\beta=\pi / 12$, $\mathrm{PA}^{\prime}$ and $\mathrm{PB}^{\prime}$ where two jets originate move upwards along the bubble surface and a downward jet (at least a dent on the bubble 
top) is also observed during the collapse phase. The first jet originates on PA' and a large vertical deviation of the jet occurs due to the Bjerknes force from the free surface. During the development of the first jet, a downward jet originates on the top of the coalesced bubble. This is closely followed by a third horizontal jet originating on $\mathrm{PB}^{\prime}$. When $\beta=\pi / 6$, the collapse pattern of the coalesced bubble is similar to that in $\beta=\pi / 12$ case. The two jets originating from PA' and PB' are more pronounced and the downward jet originating from the bubble top tends to disappear. As $\beta$ further increases, only two jets originating on PA' and PB' are formed (see frames c-f). It is worth noting that, when $\beta$ is larger than $\pi / 4$, more fluid rushes into the jet that originates on PA' and the jet tip transforms from a relatively sharp shape to a round (or flattened) shape while only a little change is observed on the shape of the second jet originating on $\mathrm{PB}$. For the influence of $\beta$ on the shape of the free surface spike, it can be seen that the spike obtains a symmetrical shape when $\beta$ equals 0 or $\pi / 2$, otherwise the movement direction of the spike deviates from the vertical direction. The most obvious deviation is achieved when $\beta=\pi / 4$.

Time histories of the centroid displacement and Kelvin impulse (defined as $I_{z}=\int_{S_{b}} \varphi^{*} \cdot n_{z} \mathrm{~d} S$ in dimensionless form) (Blake and Gibson, 1987) of the bubble system along the z-axis are plotted in Fig. 11. In Fig. 11 (a), the migration trends of the bubble centroid with different $\beta$ are almost the same. The bubble system migrates towards the free surface at first during the expansion phase and then starts to migrate downward at the later stage of the expansion phase. As $\beta$ decreases, the repulsion effect from the free surface on the bubbles becomes stronger and the centroid of the bubble system migrates downward more rapidly during the collapse phase, which is due to a larger Bjerknes force. A rapid downward migration is observed when $\beta \leq \pi / 4$. Fig. 11 (b) presents the Kelvin impulse associated with the bubble motion considered in Fig. 10. In these cases, both buoyancy force and the Bjerknes force act on the coalesced bubble. The sign of the Kelvin impulse maintains negative, which means the Bjerknes force dominates the overall process. The trends of the Kelvin impulse remain the same as $\beta$ increases. The magnitude of the Kelvin impulse increases initially and achieves its peak before the maximum volume of the coalesced bubble is attained. Afterwards, a varying trend in which the magnitude of the Kelvin impulse decreases and then increases again is observed in the collapse phase. It is noted that the buoyancy effect dominates the variation of the Kelvin impulse around the maximum bubble volume moment. The magnitude of the Kelvin impulse at the jet impact moment is larger than the first peak in $\beta \leq \pi / 4$ cases, which indicates the rapid downward migration of the bubble system. 


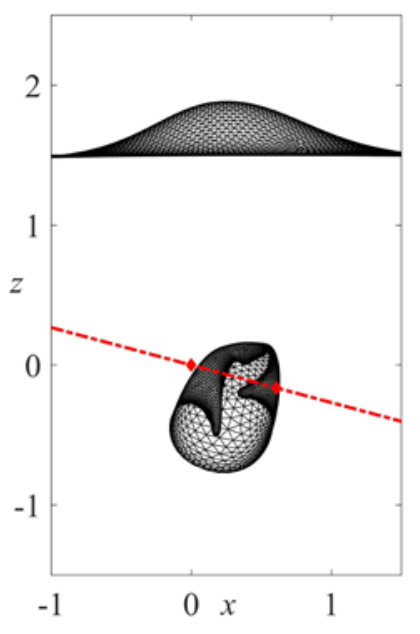

(a) $\beta=\pi / 12$

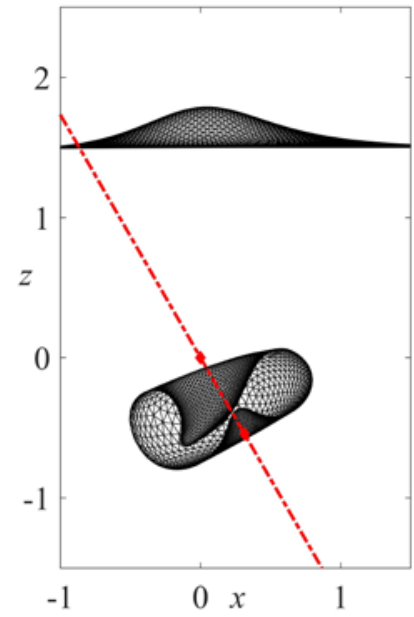

(d) $\beta=\pi / 3$

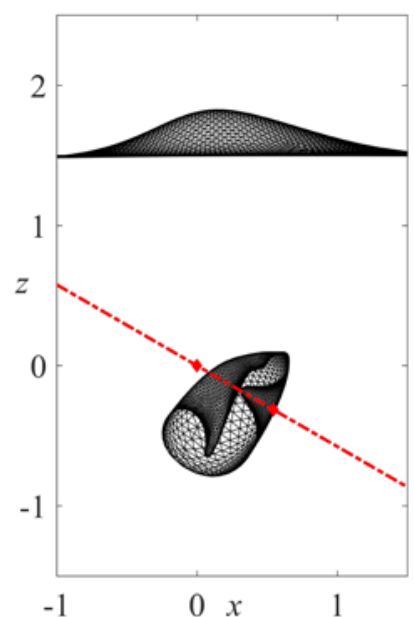

(b) $\beta=\pi / 6$

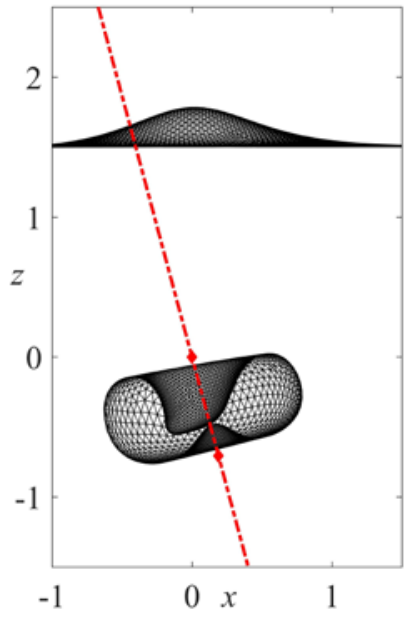

(e) $\beta=5 \pi / 12$

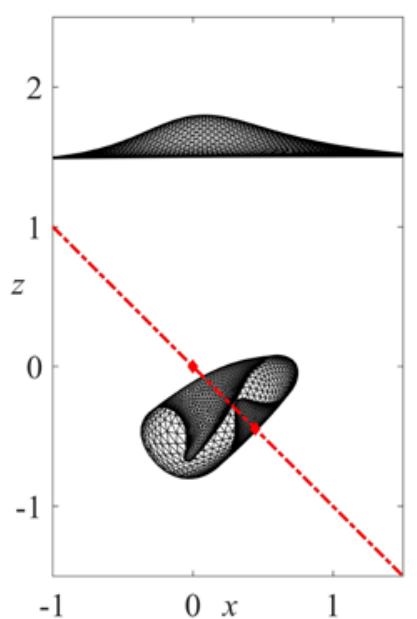

(c) $\beta=\pi / 4$

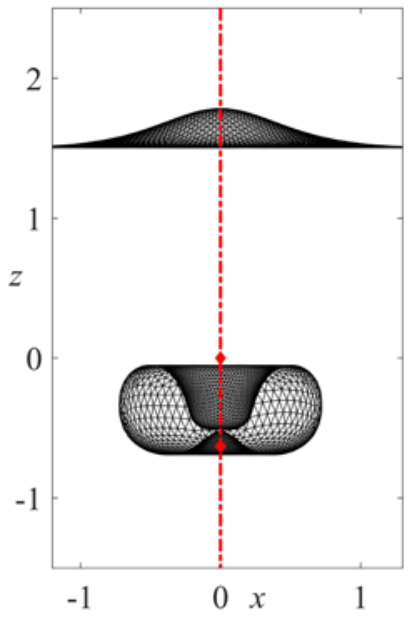

(f) $\beta=\pi / 2$

Fig. 10 Effects of the angle parameter $\beta$ on bubble and free-surface profiles at the impact moment.

Dimensionless parameters in numerical simulation are: $\gamma_{b f}=1.5, \gamma_{b b}=0.63, \alpha=1, \varepsilon=140, R_{01}=R_{02}$ $=0.1316$ and $\delta=0.22$. The angle parameters are $\beta=\pi / 12, \pi / 6, \pi / 4, \pi / 3,5 \pi / 12$ and $\pi / 2$, respectively. 


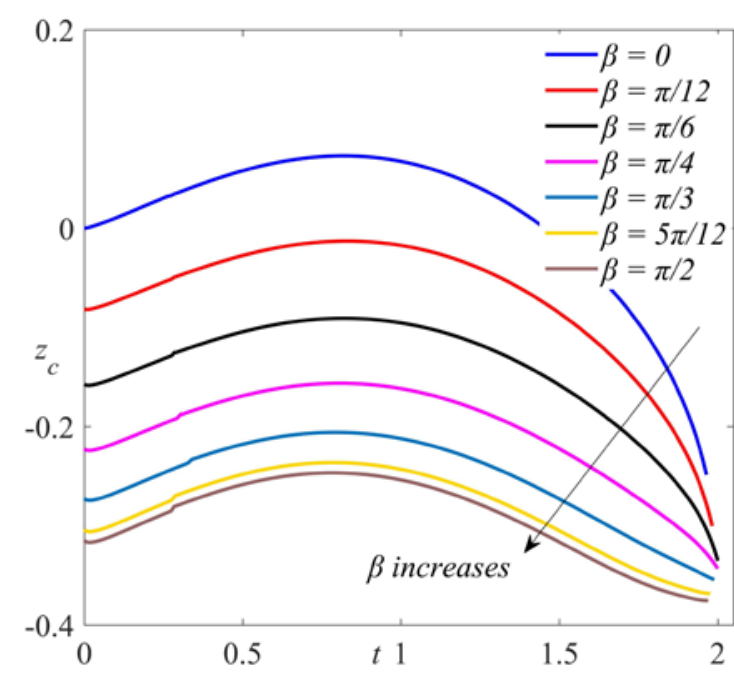

(a)

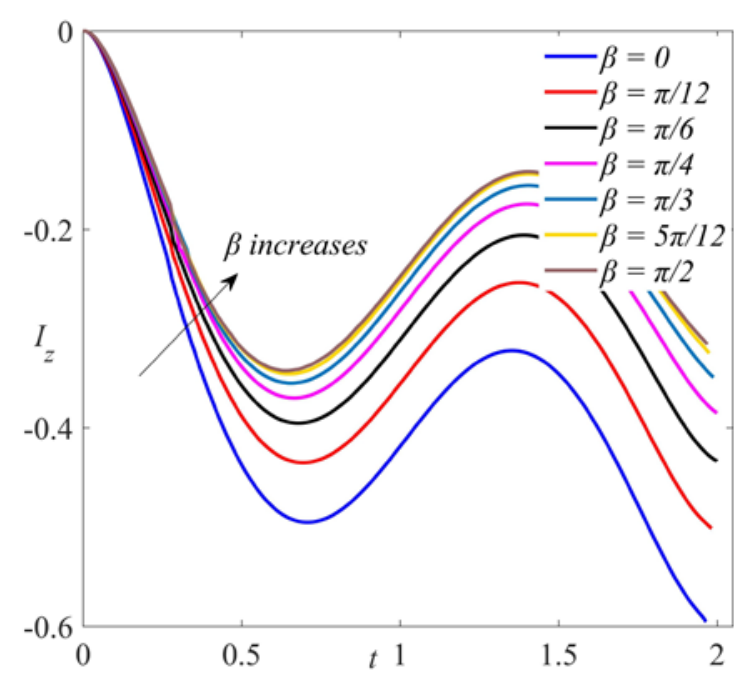

(b)

Fig. 11 Effects of the angle parameter $\beta$ on time histories of (a) the centroid displacement of the bubble system along the $z$-axis and (b) Kelvin impulse of the bubble system in $z$-direction. The distance parameters are $\gamma_{b f}=1.5$ and $\gamma_{b b}=0.63$.

In single-bubble-free-surface cases, previous studies reported that the jet formed inside a single bubble impacts on the opposite bubble surface in an annular ring when $\gamma_{b f}=1.5$ (Blake et al., 1987; Supponen et al., 2017; Wang et al., 1996b). However, the coalesced bubble beneath a free surface has quite different collapse pattern and jet formation features. To further demonstrate this with detailed analyses, the variations of the kinetic energy and the potential energy for single-bubble case are calculated and time histories of the ratio of the kinetic energy to the total energy is plotted in Fig. 12. Numerical results of a coalesced bubble for $\beta=0, \pi / 6, \pi / 3$ and $\pi / 2$ are also given for comparison. At the jet impact moment, the ratio of the kinetic energy in the single-bubble case is only $10 \%$ and a large potential energy is reserved for the subsequent violent collapse, which may lead to a relatively stronger shock wave at the minimum volume of the bubble (Supponen et al., 2017). In the present cases, however, two jets are formed during the bubble collapse phase and are associated with a large kinetic energy ( $\sim 50 \%$ of the total energy). Consequently, the collapse of the bubble is relatively weaker than that in the single-bubble case. It is further predicted that a relatively weaker shock wave would be emitted by the coalesced bubble and less energy would be radiated through shock wave to the far field. Besides, little difference is observed between two-bubble cases of different $\beta$. 


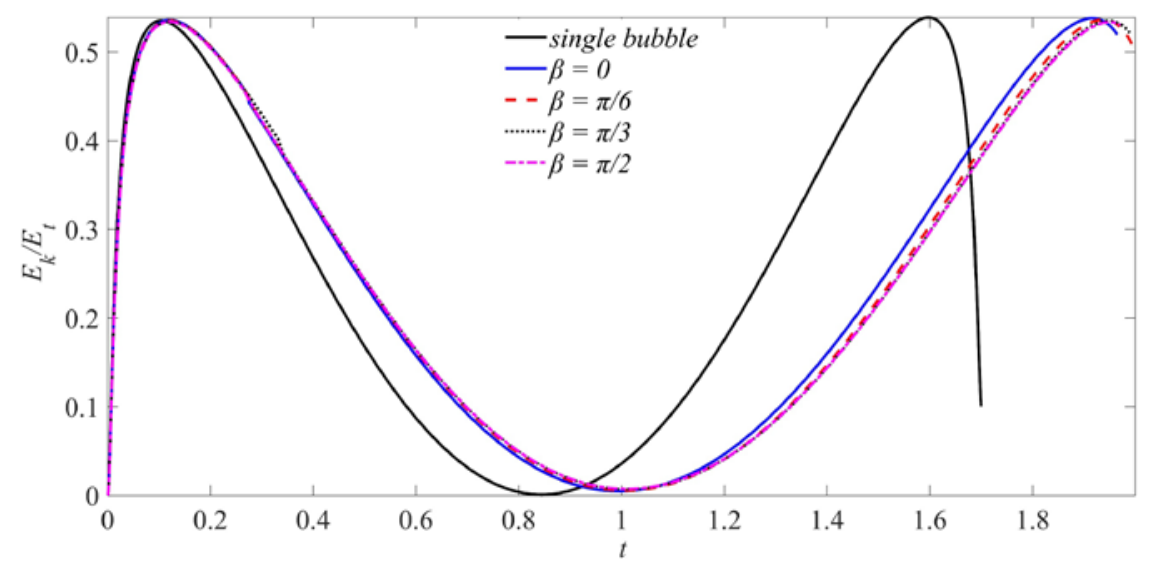

Fig. 12 Time histories of the ratio of the kinetic energy $E_{k}$ to the total energy $E_{t}$ of the system. Numerical result in single-bubble case is compared with those in the coalesced bubble cases for $\beta=$ $0, \pi / 6, \pi / 3$ and $\pi / 2$.

\subsection{Strong bubble-free-surface interaction with different $\beta$}

A series of simulations are conducted to further examine the dynamics with stronger bubble-freesurface interaction. The distance parameters remain the same as those in case 4, i.e., $\gamma_{b b}=0.73$ and $\gamma_{b f}$ $=0.85$ and the $\beta$ value ranges from 0 to $\pi / 2$. Other parameters are the same as those in Fig. 10 . Bubble profiles at the jet impact with different $\beta$ are shown in Fig. 13 and the two initial bubble centers and the connecting line are also plotted. The evolutions of the coalesced bubble and free-surface spike when $\beta=0$ are similar to those in Fig. 7 , which confirms that the strength parameter has little effect on bubble dynamic behaviors and free-surface motion and buoyancy effect is insignificant in this case. However, the strong interaction makes the physical phenomena quite different from those with $\gamma_{b f}=$ 1.5. For $\beta=\pi / 12$, a downward jet originating on the top of the coalesced bubble is formed at first. During the development of the first jet, another downward jet is formed at the base of the first jet (shown by the red arrow in frame a). Subsequently, a third jet is gradually formed at the base of the first jet (shown by the blue arrow in frame a). Finally, the right protrusion on the main jet is about to impact on the right bubble surface. The shape of the bubble jet in this case appears to have several corners, which needs further validation in future experimental studies. For $\beta=\pi / 6$, similar features are observed and the first main jet is about to penetrate the coalesced bubble, as shown in frame (b). The jet inside the bubble obtains a larger volume than that in frame (a). As $\beta$ further increases ( $\pi / 4 \leq \beta<$ $\pi / 2$ ), the second and third jets tend to disappear and only an oblique jet is observed. For $\beta=\pi / 2$, only 
a downward jet is formed inside the coalesced bubble. As for the free-surface spike, the shape of the spike clearly changes with $\beta$. When $\beta=0$ (shown in Fig. 7), a broad spike is observed at the jet impact moment in the front view. When $\beta=\pi / 12$, an oblique spike is observed and a bump appears on the right-hand side of the spike. The bump gradually disappears with increasing $\beta$ and a thin spike in symmetric shape is obtained when $\beta=\pi / 2$.

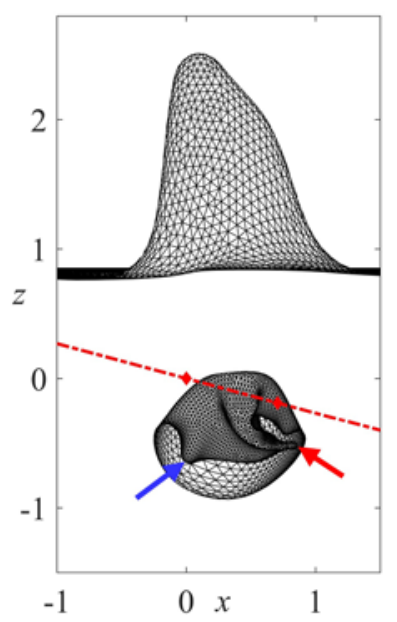

(a) $\beta=\pi / 12$

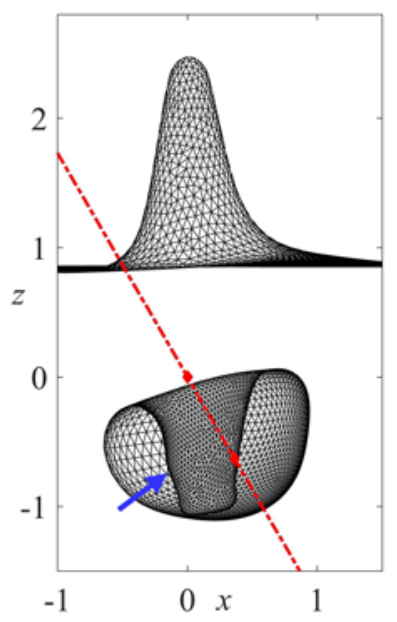

(d) $\beta=\pi / 3$

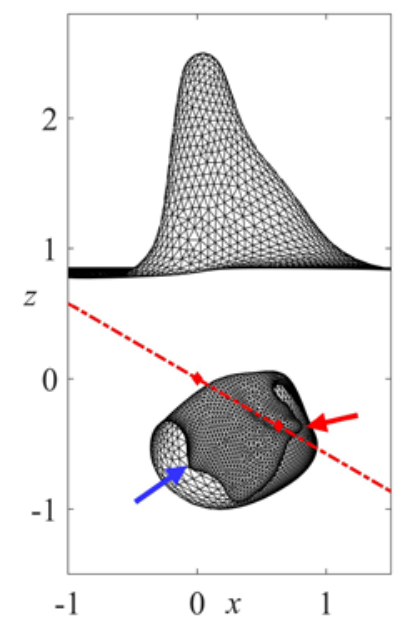

(b) $\beta=\pi / 6$

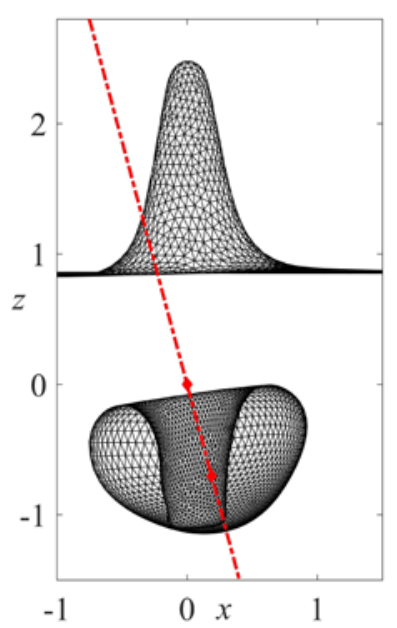

(e) $\beta=5 \pi / 12$

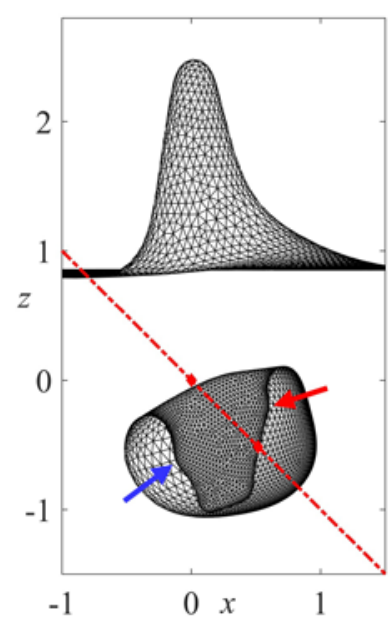

(c) $\beta=\pi / 4$

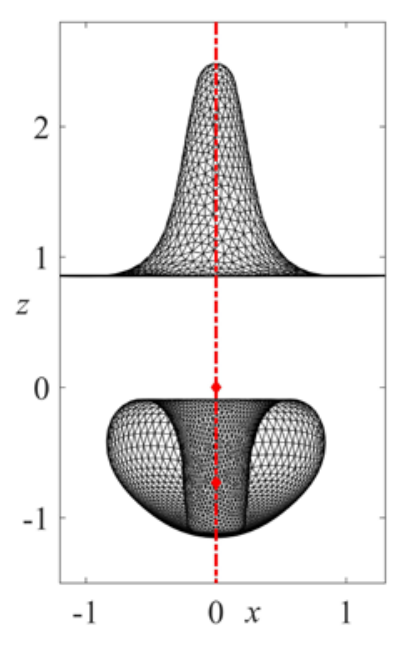

(f) $\beta=\pi / 2$

Fig. 13 Bubble and free-surface profiles at the jet impact moment with different $\beta$. Dimensionless parameters in numerical simulation are: $\gamma_{b f}=0.85, \gamma_{b b}=0.73, \alpha=1, \varepsilon=140, R_{01}=R_{02}=0.1316$ and $\delta=0.22$. The angle parameters are $\beta=\pi / 12, \pi / 6, \pi / 4, \pi / 3,5 \pi / 12$ and $\pi / 2$, respectively.

Time histories of the centroid displacement and Kelvin impulse of the bubble system along the zaxis with different $\beta$ are plotted in Fig. 14. As shown in Fig. 14 (a), the centroid displacement of the 
bubble system along the $z$-axis increases almost linearly at first and the increase speeds are almost the same for different $\beta$. Then the bubble system is repelled by the free surface in the late expansion phase. The downward migration magnitude of the bubble system decreases with $\beta$. For $\beta \geq \pi / 3$, the effect of $\beta$ on the bubble migration is negligible. As shown in Fig. 14 (b), the magnitude of the Kelvin impulse is more than three times of that in Fig. 11 (b) as $\gamma_{b f}$ is reduced from 1.5 to 0.85 indicating that the Bjerknes force from the free surface dominates the variation of the Kelvin impulse in such small $\gamma_{b f}$ cases.
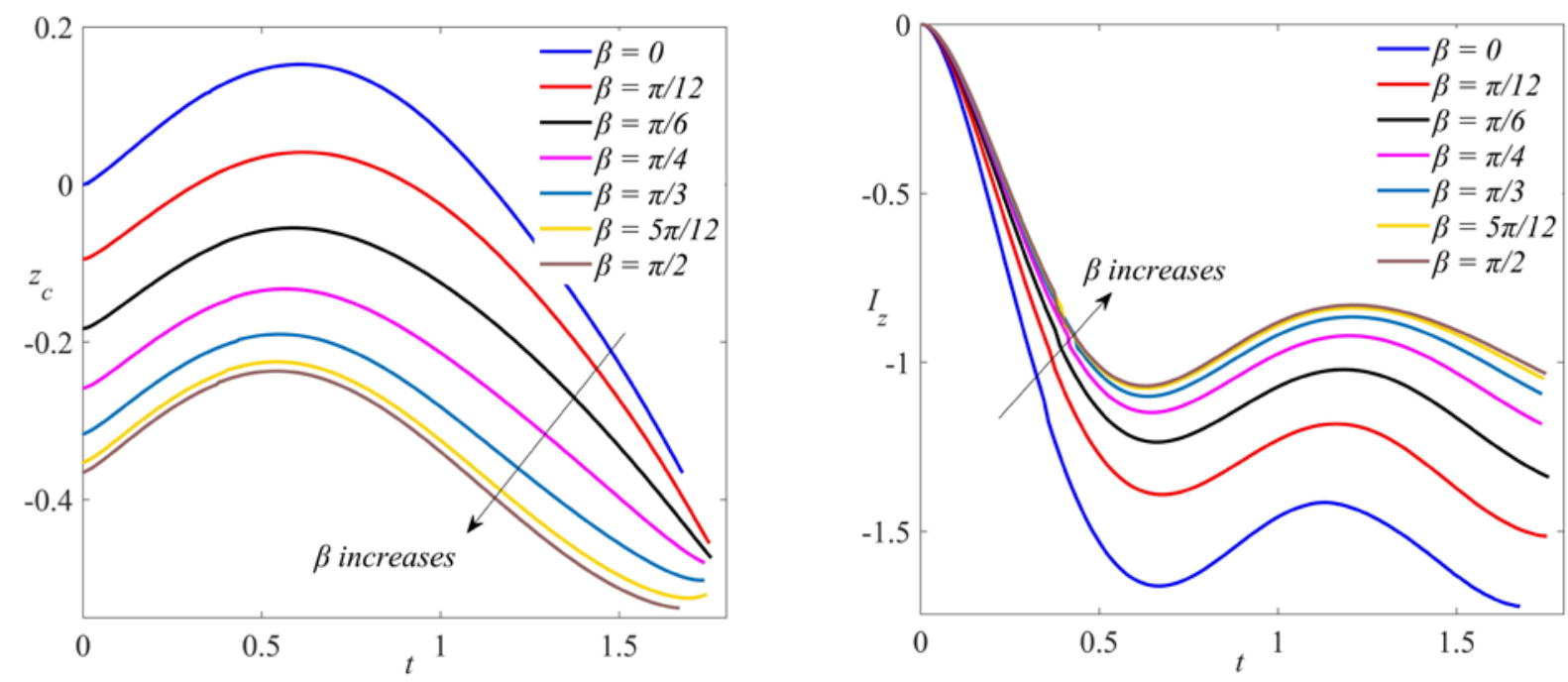

Fig. 14 Effects of the angle parameter $\beta$ on time histories of (a) the centroid displacement of the bubble system along the z-axis and (b) Kelvin impulse of the bubble system in the z-direction. The distance parameters are kept the same as in case 4, i.e. $\gamma_{b f}=0.85$ and $\gamma_{b b}=0.73$.

\subsection{Further remarks}

The maximum bubble volume $V_{\max }$ is an important characteristic parameter in bubble dynamics, which is often used to estimate the bubble energy and bubble period (Lee et al., 2007). The variations of $V_{\max }$ and the time when the maximum volume is attained $t_{\max }$ (approximately half of the bubble period) versus $\beta$ are presented in Fig. 15. Different trends are observed for $\gamma_{b f}=1.5$ and $\gamma_{b f}=0.85$. For $\gamma_{b f}=1.5$, the maximum volume of the coalesced bubble $V_{\max }$ decreases with $\beta$, due to the fact that a higher hydrostatic pressure makes the bubble attain a smaller maximum volume. However, the change in $t_{\max }$ versus $\beta$ is below $0.65 \%$ of the bubble period indicating that the angle parameter $\beta$ has little effect on the time when the maximum volume is attained $t_{\max }$. If a single bubble oscillates in an infinite 
flow field or beneath a free surface $\left(\gamma_{b f}=1.5\right), t_{\max }$ is 0.988 and 0.847 , respectively. Comparing the motion of a single bubble with and without a free surface, the weak bubble-free-surface interaction shortens the bubble period by $14.3 \%$; while in the two-bubble cases, the existence of another bubble and the coalescence make $t_{\max }$ larger than 1 and lead to a longer bubble period. When $\gamma_{b f}=0.85$, the peak $V_{\max }$ is obtained at $\beta=\pi / 12$ and $V_{\max }$ decreases rapidly with $\beta$. In the strong interaction cases, $t_{\max }$ increases rapidly at first with $\beta$ and then approaches to a constant for $\beta \geq \pi / 4$. During the interaction between a single bubble and a free surface $\left(\gamma_{b f}=0.85\right), t_{\max }$ is only 0.735 which is shortened by $25.6 \%$ compared with the motion of a single bubble in an infinite field. In the two-bubble cases, $t_{\max }$ becomes larger than that in the single-bubble-free-surface interaction, but is still shorter than that of a single bubble in an infinite field. Though coalescence of two bubbles occurs in the expansion phase, the strong effect of the free surface shortens the bubble period greatly and the maximum $t_{\max }$ is still $10.4 \%$ smaller than that in the single bubble case.
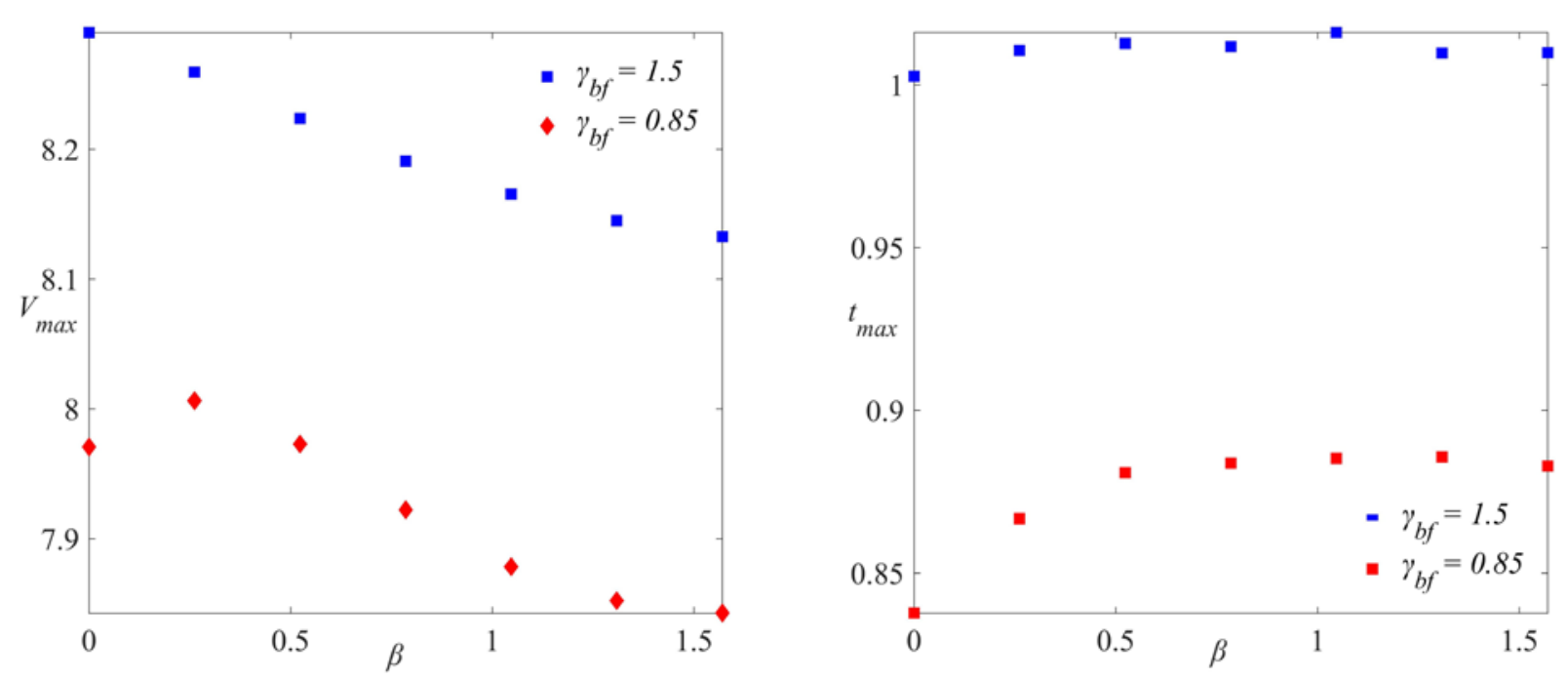

Fig. 15 Effects of the angle parameter $\beta$ on (a) the maximum volume of the coalesced bubble $V_{\max }$ and (b) the time when the maximum volume is attained $t_{\max }$ for $\gamma_{b f}=1.5$ and $\gamma_{b f}=0.85$. The angle parameter ranges from 0 to $\pi / 2$ with an interval of $\pi / 12$.

The above laws are useful to control the bubble oscillation period in practical applications. For example, in the seabed exploration field, the airguns should not be placed at very small water depth. Although the ambient hydrostatic pressure reduces with decreasing water depth, the strong free surface effect shortens the bubble period significantly, especially when the dimensionless water depth $\gamma_{b f}$ is less than 1 . On the other hand, the bubble survives fewer cycles with a smaller water depth because of 
the bubble bursting effect (Wang et al., 2015b). Besides, in order to obtain a relatively lower frequency sound waves in seabed exploration, a longer bubble period is desirable in airgun operations. This can be easily done by using the "two-bubble coalescence". By adjusting the governing parameters, a specific bubble period can be achieved. If two bubbles are not enough, maybe more bubbles are needed. The interaction and coalescence of multiple bubbles will be for our future work.

Time histories of the height of the free-surface spike for $\gamma_{b f}=1.5$ and $\gamma_{b f}=0.85$ are shown in Fig. 16 and the trends are markedly different. In weak interaction cases (see Fig. 16(a)), the height of the free-surface spike increases rapidly in the early expansion phase followed by a slowing down of the upward motion of the spike. In the collapse phase, the upward velocity of the spike gradually decreases to zero and then the free-surface spike moves downward slowly, which is mainly induced by the attraction of the collapsing bubble and the gravity effect plays a minor role in this process. The maximum height of the free-surface spike is obtained at $\beta=0$. When $\gamma_{b f}=0.85$, the height of the freesurface spike increases rapidly in the early expansion phase and then the upward velocity decreases, but the spike height still increases almost linearly. The height of the free-surface spike is the largest at $\beta=0$ and only slight differences are observed for $\beta>0$, which indicates that the increase of $\beta$ slightly reduces the effect of the bubble motion on the free surface. Various applications can benefit from the findings, for instance, the spike motion is associated with applications of laser induced forward transfer (LIFT) printing (Turkoz et al., 2018b), and the spike (a wall of water) is also a low cost, universal terminal defense system to protect Navy ships from high-speed, sea-skimming, antiship cruise missiles (Connor and Higdon, 1996).
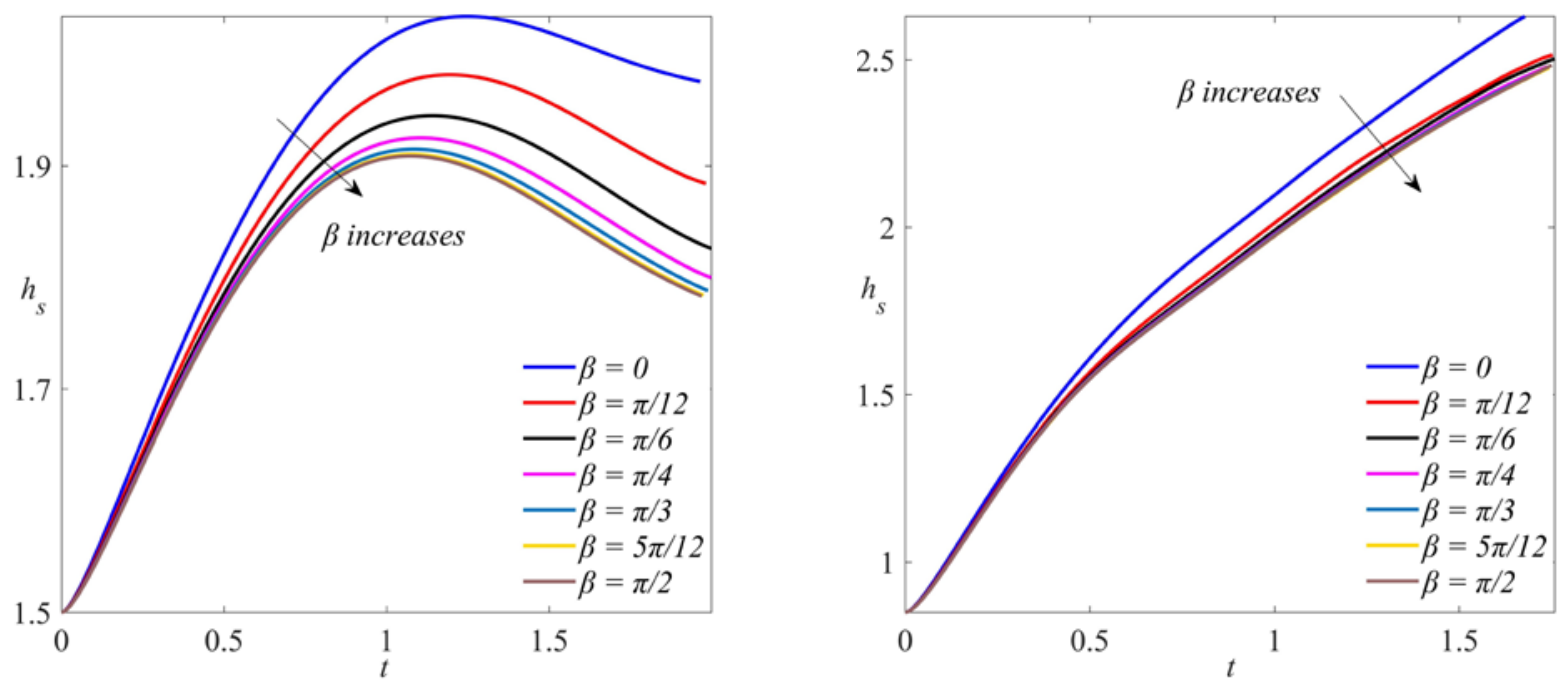

Fig. 16 Effects of the angle parameter $\beta$ on the height of the free-surface spike for (a) $\gamma_{b f}=1.5$ and 
(b) $\gamma_{b f}=0.85$. The angle parameter ranges from 0 to $\pi / 2$ with an interval of $\pi / 12$.

\section{CONCLUSIONS}

The strong interaction between two oscillating bubbles and a free surface was studied to investigate the pre-coalescence and post-coalescence of two bubbles, and the free-surface evolution. The underwater electric discharge method was adopted to generate two similar sized bubbles beneath a free surface, captured by a high speed camera. Both axiymmetric and three-dimensional BIM models were established and several numerical techniques were implemented in the present 3D model. For axisymmetric-configuration cases, good agreement between the two models was achieved, indicating the high accuracy of the 3D model. The main features of several representative experiments in 3D configurations were well reproduced by the 3D model with more detailed numerical results.

For the two axisymmetric-configuration cases $(\beta=\pi / 2)$ in this study, the jetting behavior of the coalesced bubble is mainly affected by the free surface, only a downward jet is observed. For the $\gamma_{b f}=$ 0.74 case, a very strong interaction between the coalesced bubble and the free surface is observed, and the tip of the thin liquid jet is flattened and quite unstable at the rim, which is companied with the splashing and splitting of tiny droplets. Following the jet penetrating, a protrusion forms at the bottom of the bubble and subsequently the splitting is observed at the intersection between the protrusion and the main bubble.

For two bubbles arranged in a horizontal line, the dynamic process of bubble coalescence exhibits distinct different features due to the effect of free surface. If the effect of the free surface is relatively weak (i.e., $\gamma_{b f}=1.51$ ), three jets are generated during the bubble collapse phase, two of which originate on the upper left and upper right surfaces of the coalesced bubble. Afterwards, a downward jet with a flattened tip originates on the top of the coalesced bubble. The three jets squeeze each other and may collide inside the coalesced bubble. If the effect of the free surface is relatively strong (i.e., $\gamma_{b f}=0.85$ ), the downward jet is more squeezed by the two oblique jets; thus a sheet-like liquid jet is gradually formed with a large width in side view. There are two contact points at the jet impact moment.

For two bubbles arranged in an oblique configuration beneath a free surface, the downward jet and the secondary jet squeezes each other during the collapse phase. A distinct shape of the bubble jet at the impact moment is observed with several corners. An oblique spike is also observed in the 
experiment and captured by the present numerical simulation.

The effects of the distance parameter $\gamma_{b f}$ and the angle parameter $\beta$ are investigated. As $\gamma_{b f}$ decreases, both the maximum volume of the coalesced bubble $V_{\max }$ and the bubble period decrease, and the upward jet tends to disappear if the bubble is subject to buoyancy. If the bubble-free-surface interaction is relatively weak (i.e., $\gamma_{b f}=1.5$ ), the maximum volume of the coalesced bubble $V_{\max }$ decreases with $\beta$. However, if the bubble-free-surface interaction is relatively strong (i.e., $\gamma_{b f}=0.85$ ), a more complex trend is observed, namely, $V_{\max }$ increases first and then decreases with $\beta$, the bubble period increases rapidly at first with $\beta$ and approaches to a constant for $\beta \geq \pi / 4$. As $\gamma_{b f}$ increases, the effect of $\beta$ on bubble period decreases. For $\gamma_{b f}=1.5$, the change in dependence of the bubble period on $\beta$ is less than $0.65 \%$. The increase of $\beta$ weakens the free surface motion.

Compared with single bubble situations, the collapsing coalesced bubble beneath a free surface possesses a much higher kinetic energy, which is associated with the energetic jet. It is reckoned that a relatively weaker shock wave would be emitted by the coalesced bubble and thus less energy would be radiated outward.

\section{ACKNOWLEDGMENTS}

This work is supported by the National Key R\&D Program of China (2018YFC0308900), the National Natural Science Foundation of China (11702071 and 51709056), the China Postdoctoral Science Foundation (2017M620112, 2018T110276 and 2017M621249) and the Heilongjiang Postdoctoral Fund (LBH-Z17049).

\section{References}

Best, J., 1993. The formation of toroidal bubbles upon the collapse of transient cavities. Journal of Fluid Mechanics 251, 79-107.

Best, J., Kucera, A., 1992. A numerical investigation of non-spherical rebounding bubbles. Journal of Fluid Mechanics 245, 137-154.

Blake, J., Taib, B., Doherty, G., 1987. Transient cavities near boundaries Part 2. Free surface. Journal of Fluid Mechanics 181, 197-212.

Blake, J.R., Gibson, D., 1987. Cavitation bubbles near boundaries. Annual Review of Fluid Mechanics 19 (1), 99-123.

Bremond, N., Arora, M., Dammer, S.M., Lohse, D., 2006a. Interaction of cavitation bubbles on a wall. Physics of Fluids 18 (12), 121505. 
Bremond, N., Arora, M., Ohl, C.D., Lohse, D., 2006b. Controlled multibubble surface cavitation. Physical Review Letters 96 (22), 224501.

Brett, J.M., Krelle, A., 2018. A study of bubble collapse pressure pulse waves from small scale underwater explosions near the water surface. Journal of Sound and Vibration 435, 91-103.

Brujan, E.-A., Noda, T., Ishigami, A., Ogasawara, T., Takahira, H., 2018. Dynamics of laser-induced cavitation bubbles near two perpendicular rigid walls. Journal of Fluid Mechanics 841, 28-49.

Chen, H., Li, X., Wan, M., Wang, S., 2009. High-speed observation of cavitation bubble clouds near a tissue boundary in high-intensity focused ultrasound fields. Ultrasonics 49 (3), 289-292.

Chew, L.W., Klaseboer, E., Ohl, S.-W., Khoo, B.C., 2011. Interaction of two differently sized oscillating bubbles in a free field. Physical Review E 84 (6), 066307.

Chew, L.W., Klaseboer, E., Ohl, S.-W., Khoo, B.C., 2013. Interaction of two oscillating bubbles near a rigid boundary. Experimental Thermal and Fluid Science 44, 108-113.

Connor, J.G., Higdon, C.E., 1996. Water Barrier Line Charge Plume Video Analysis. DTIC Document. Cox, E., Pearson, A., Blake, J.R., Otto, S.R., 2004. Comparison of methods for modelling the behaviour of bubbles produced by marine seismic airguns. Geophysical Prospecting 52 (5), 451-477.

Cui, P., Wang, Q.X., Wang, S.P., Zhang, A.M., 2016a. Experimental study on interaction and coalescence of synchronized multiple bubbles. Physics of Fluids 28, 012103.

Cui, P., Zhang, A.M., Wang, S.P., 2016b. Small-charge underwater explosion bubble experiments under various boundary conditions. Physics of Fluids 28, 117103.

Curtiss, G.A., Leppinen, D.M., Wang, Q.X., Blake, J.R., 2013. Ultrasonic cavitation near a tissue layer. Journal of Fluid Mechanics 730, 245-272.

Dadvand, A., Khoo, B.C., Shervani-Tabar, M.T., Khalilpourazary, S., 2012. Boundary element analysis of the droplet dynamics induced by spark-generated bubble. Engineering Analysis with Boundary Elements 36 (11), 1595-1603.

de Graaf, K.L., Penesis, I., Brandner, P.A., 2014. Modelling of seismic airgun bubble dynamics and pressure field using the Gilmore equation with additional damping factors. Ocean Engineering 76, 3239.

Fong, S.W., Adhikari, D., Klaseboer, E., Khoo, B.C., 2009. Interactions of multiple spark-generated bubbles with phase differences. Experiments in Fluids 46 (4), 705-724.

Han, R., Li, S., Zhang, A., Wang, Q., 2016. Modelling for three dimensional coalescence of two bubbles. Physics of Fluids 28 (6), 062104.

Han, R., Zhang, A.M., Li, S., 2014. Three-dimensional numerical simulation of crown spike due to coupling effect between bubbles and free surface. Chinese Physics B 23 (3), 034703.

Han, R., Zhang, A.M., Li, S., Zong, Z., 2018. Experimental and numerical study of the effects of a wall on the coalescence and collapse of bubble pairs. Physics of Fluids 30 (4), 042107.

Ji, C., Li, B., Zou, J., Yang, H., 2017. Interaction of two spark generated bubbles beneath free surface. Experimental Thermal and Fluid Science 81, 76-83.

Kannan, Y.S., Karri, B., Sahu, K.C., 2018. Letter: Entrapment and interaction of an air bubble with an oscillating cavitation bubble. Physics of Fluids 30 (4), 041701.

Klaseboer, E., Hung, K.C., Wang, C., Wang, C.W., Khoo, B.C., Boyce, P., Debono, S., Charlier, H., 2005a. Experimental and numerical investigation of the dynamics of an underwater explosion bubble near a resilient/rigid structure. Journal of Fluid Mechanics 537, 387-413.

Klaseboer, E., Khoo, B.C., Hung, K.C., 2005b. Dynamics of an oscillating bubble near a floating structure. Journal of Fluids and Structures 21 (4), 395-412.

Koukouvinis, P., Gavaises, M., Supponen, O., Farhat, M., 2016. Simulation of bubble expansion and 
collapse in the vicinity of a free surface. Physics of Fluids 28 (5), 052103.

Lee, M., Klaseboer, E., Khoo, B.C., 2007. On the boundary integral method for the rebounding bubble. Journal of Fluid Mechanics 570, 407.

Li, S., Khoo, B.C., Zhang, A.M., Wang, S., 2018a. Bubble-sphere interaction beneath a free surface. Ocean Engineering 169, 469-483.

Li, S., Ni, B., 2016. Simulation on the interaction between multiple bubbles and free surface with viscous effects. Engineering Analysis with Boundary Elements 68, 63-74.

Li, S., Zhang, A.-M., Han, R., Ma, Q., 2019. 3D full coupling model for strong interaction between a pulsating bubble and a movable sphere. Journal of Computational Physics 392, 713-731.

Li, S., Zhang, A.-M., Wang, S., Han, R., 2018b. Transient interaction between a particle and an attached bubble with an application to cavitation in silt-laden flow. Physics of Fluids 30 (8), 082111.

Li, S., Zhang, A.M., Han, R., Liu, Y.Q., 2017. Experimental and numerical study on bubble-sphere interaction near a rigid wall. Physics of Fluids 29 (9), 092102.

Liu, N.N., Cui, P., Ren, S.F., Zhang, A.M., 2017. Study on the interactions between two identical oscillation bubbles and a free surface in a tank. Physics of Fluids 29 (5), 052104.

Liu, N.N., Cui, Y.D., Khoo, B.C., Zhang, A.M., 2018. Damage characteristics of elastic material through a thin membrane using high-intensity focused ultrasound (HIFU). AIP Advances 8 (11), 115123.

Liu, Y.L., Wang, Q.X., Wang, S.P., Zhang, A.M., 2016. The motion of a 3D toroidal bubble and its interaction with a free surface near an inclined boundary. Physics of Fluids 28 (12).

Long, X., Cheng, H., Ji, B., Arndt, R.E.A., Peng, X., 2018. Large eddy simulation and EulerLagrangian coupling investigation of the transient cavitating turbulent flow around a twisted hydrofoil. International Journal of Multiphase Flow 100, 41-56.

Lundgren, T.S., Mansour, N.N., 1988. Oscillations of drops in zero gravity with weak viscous effects. Journal of Fluid Mechanics 194, 479-510.

Ochiai, N., Ishimoto, J., 2017. Numerical investigation of multiple-bubble behaviour and induced pressure in a megasonic field. Journal of Fluid Mechanics 818, 562-594.

Pearson, A., Cox, E., Blake, J.R., Otto, S.R., 2004. Bubble interactions near a free surface. Engineering Analysis with Boundary Elements 28 (4), 295-313.

Quah, E.W., Karri, B., Ohl, S.-W., Klaseboer, E., Khoo, B.C., 2018. Expansion and collapse of an initially off-centered bubble within a narrow gap and the effect of a free surface. International Journal of Multiphase Flow 99, 62-72.

Robinson, P.B., Blake, J.R., Kodama, T., Shima, A., Tomita, Y., 2001. Interaction of cavitation bubbles with a free surface. Journal of Applied Physics 89 (12), 8225.

Rosselló, J.M., Lauterborn, W., Koch, M., Wilken, T., Kurz, T., Mettin, R., 2018. Acoustically induced bubble jets. Physics of Fluids 30 (12), 122004.

Rungsiyaphornrat, S., Klaseboer, E., Khoo, B., Yeo, K., 2003. The merging of two gaseous bubbles with an application to underwater explosions. Computers \& Fluids 32 (8), 1049-1074.

Saleki-Haselghoubi, N., Shervani-Tabar, M.T., Taeibi-Rahni, M., Dadvand, A., 2016. Interaction of two spark-generated bubbles near a confined free surface. Theoretical and Computational Fluid Dynamics 30 (3), 185-209.

Supponen, O., Obreschkow, D., Kobel, P., Tinguely, M., Dorsaz, N., Farhat, M., 2017. Shock waves from nonspherical cavitation bubbles. Physical Review Fluids 2 (9), 093601.

Tagawa, Y., Peters, I.R., 2018. Bubble collapse and jet formation in corner geometries. Physical Review Fluids 3 (8), 081601. 
Turangan, C., Ong, G., Klaseboer, E., Khoo, B., 2006. Experimental and numerical study of transient bubble-elastic membrane interaction. Journal of Applied Physics 100 (5), 054910.

Turkoz, E., Kang, S., Deike, L., Arnold, C.B., 2018a. Subthreshold laser jetting via flow-focusing in laser-induced forward transfer. Physical Review Fluids 3 (8), 082201.

Turkoz, E., Perazzo, A., Kim, H., Stone, H.A., Arnold, C.B., 2018b. Impulsively Induced Jets from Viscoelastic Films for High-Resolution Printing. Physical Review Letters 120 (7), 074501.

Verschoof, R.A., van der Veen, R.C.A., Sun, C., Lohse, D., 2016. Bubble Drag Reduction Requires Large Bubbles. Physical Review Letters 117 (10), 104502.

Wang, C., Khoo, B.C., 2004. An indirect boundary element method for three-dimensional explosion bubbles. Journal of Computational Physics 194 (2), 451-480.

Wang, C., Khoo, B.C., Yeo, K.S., 2003. Elastic mesh technique for 3D BIM simulation with an application to underwater explosion bubble dynamics. Computers \& Fluids 32 (9), 1195-1212.

Wang, J.X., Zong, Z., Liu, K., Cui, J., 2018a. Simulations of the dynamics and interaction between a floating structure and a near-field explosion bubble. Applied Ocean Research 78, 50-60.

Wang, Q., Manmi, K., Calvisi, M.L., 2015a. Numerical modeling of the 3D dynamics of ultrasound contrast agent microbubbles using the boundary integral method. Physics of Fluids 27 (2), 022104.

Wang, Q.X., 2004. Numerical simulation of violent bubble motion. Physics of Fluids 16 (5), 1610.

Wang, Q.X., Manmi, K., 2014. Three dimensional microbubble dynamics near a wall subject to high intensity ultrasound. Physics of Fluids 26 (3), 032104.

Wang, Q.X., Yeo, K.S., Khoo, B.C., Lam, K.Y., 1996a. Nonlinear interaction between gas bubble and free surface. Computers \& Fluids 25 (7), 607-628.

Wang, Q.X., Yeo, K.S., Khoo, B.C., Lam, K.Y., 1996b. Strong interaction between a buoyancy bubble and a free surface. Theoretical and Computational Fluid Dynamics 8 (1), 73-88.

Wang, S., Duan, W., Wang, Q., 2015b. The bursting of a toroidal bubble at a free surface. Ocean Engineering 109, 611-622.

Wang, Y., Xu, C., Wu, X., Huang, C., Wu, X., 2017. Ventilated cloud cavitating flow around a blunt body close to the free surface. Physical Review Fluids 2 (8), 084303.

Wang, Z., Huang, B., Zhang, M., Wang, G., Ji, B., 2018b. Numerical investigation of ventilated cavitating vortex shedding over a bluff body. Ocean Engineering 159, 129-138.

Zhang, A., Cui, P., Cui, J., Wang, Q., 2015a. Experimental study on bubble dynamics subject to buoyancy. Journal of Fluid Mechanics 776, 137-160.

Zhang, A., Li, S., Cui, J., 2015b. Study on splitting of a toroidal bubble near a rigid boundary. Physics of Fluids 27 (6), 062102.

Zhang, A., Liu, Y., 2015. Improved three-dimensional bubble dynamics model based on boundary element method. Journal of Computational Physics 294, 208-223.

Zhang, N., Zong, Z., 2011. The effect of rigid-body motions on the whipping response of a ship hull subjected to an underwater bubble. Journal of Fluids and Structures 27 (8), 1326-1336.

Zhang, S., Wang, S.P., Zhang, A.M., Cui, P., 2018. Numerical study on motion of the air-gun bubble based on boundary integral method. Ocean Engineering 154, 70-80.

Zhang, Y.L., Yeo, K.S., Khoo, B.C., Wang, C., 2001. 3D Jet Impact and Toroidal Bubbles. Journal of Computational Physics 166 (2), 336-360.

Zong, Z., Wang, J.X., Zhou, L., Zhang, G.Y., 2015. Fully nonlinear 3D interaction of bubble dynamics and a submerged or floating structure. Applied Ocean Research 53, 236-249. 